\title{
Passive technologies for future large-scale photonic integrated circuits on silicon: polarization handling, light non-reciprocity and loss reduction
}

\begin{abstract}
Daoxin Dai ${ }^{1,2}$, Jared Bauters ${ }^{1}$ and John E Bowers ${ }^{1}$
Silicon-based large-scale photonic integrated circuits are becoming important, due to the need for higher complexity and lower cost for optical transmitters, receivers and optical buffers. In this paper, passive technologies for large-scale photonic integrated circuits are described, including polarization handling, light non-reciprocity and loss reduction. The design rule for polarization beam splitters based on asymmetrical directional couplers is summarized and several novel designs for ultra-short polarization beam splitters are reviewed. A novel concept for realizing a polarization splitter-rotator is presented with a very simple fabrication process. Realization of silicon-based light non-reciprocity devices (e.g., optical isolator), which is very important for transmitters to avoid sensitivity to reflections, is also demonstrated with the help of magneto-optical material by the bonding technology. Low-loss waveguides are another important technology for large-scale photonic integrated circuits. Ultra-low loss optical waveguides are achieved by designing a $\mathrm{Si}_{3} \mathrm{~N}_{4}$ core with a very high aspect ratio. The loss is reduced further to $<0.1 \mathrm{~dB} \mathrm{~m}^{-1}$ with an improved fabrication process incorporating a high-quality thermal oxide upper cladding by means of wafer bonding. With the developed ultra-low loss $\mathrm{Si}_{3} \mathrm{~N}_{4}$ optical waveguides, some devices are also demonstrated, including ultra-high-Q ring resonators, low-loss arrayed-waveguide grating (de)multiplexers, and high-extinction-ratio polarizers.
\end{abstract}

Light: Science \& Applications (2012) 1, e1; doi:10.1038/lsa.2012.1; published online 29 March 2012

Keywords: integration; low loss; non-reciprocal; polarization; silicon

\section{INTRODUCTION}

Large-scale photonic integrated circuits (LS-PICs) are becoming very promising for many applications, ${ }^{1,2}$ including next-generation optical networks, optical interconnects, wavelength division multiplexed systems, coherent transceivers, lab-on-chip, etc. This is because LS-PICs provide advantages, e.g., higher performance, reduced device footprint, lower component-to-component coupling losses, lower package costs and lower power consumption. However, the progress on LS-PICs is still quite limited. One reason is that the material system for photonics is much more complex than electronics. Currently, the focus is on silicon and InP substrate technologies. ${ }^{3}$ InP is a good platform for LS-PICs, since it enables both passive and active components in the same substrate. ${ }^{4}$ One recent demonstration is an $8 \times 8 \mathrm{InP}$ monolithic tunable optical router packet forwarding chip, which successfully integrated about 200 functional elements in a chip with a size of $4.25 \times 14.5 \mathrm{~mm}^{2}$ Infinera plans to deploy a $400-\mathrm{Gb} \mathrm{s}^{-1}$ PIC transmitter with more than 300 optical functions in live networks. ${ }^{5}$

On the other hand, silicon-based PICs ${ }^{6-16}$ have also become very popular, because of their compatibility with mature CMOS (complementary metal-oxide-semiconductor) technologies with excellent processing control, low cost and high volume processing. Furthermore, silicon-on-insulator (SOI) nanowires enable ultra-sharp bends (e.g., $1-2 \mu \mathrm{m}$ ), because of their ultra-high refractive index contrast. ${ }^{11}$ Consequently various ultrasmall SOI nanowire-based devices, e.g., arrayed-waveguide gratings (AWG),${ }^{7-9}$ microrings, ${ }^{10}$ etc., can be realized. However, since silicon is not a direct bandgap semiconductor material, it is not a good option for active PICs with lasers, as well as photodetectors. Fortunately, several technologies exist to solve the issue. For example, the hybrid III-V/Si platform provides a very promising solution for realizing integrated active photonic devices on silicon. ${ }^{3,12}$ Therefore, it is becoming feasible to realize LS-PICs on a silicon substrate. Examples include integrated transmitter, ${ }^{13}$ on-chip optical buffers ${ }^{14}$ and coherent optical receivers. ${ }^{15}$ However, one should note that there are still several essential issues when regarding the realization of silicon LS-PICs.

First, it is well known that SOI nanowire PICs are usually severely polarization-sensitive, because of the huge structural birefringence. ${ }^{8}$ Although it is often possible to diminish the polarization dependence for some components by using specific approaches, ${ }^{17,18}$ a general solution for the polarization issue is using a polarization diversity system consisting of polarization beam splitters (PBSs) and polarization rotators (PRs) ${ }^{6,10}$ Polarization diversity is also very important for many other applications, such as coherent receivers, as well as polarization multiplexing technologies ${ }^{19}$ for polarization-multiplexed 
transmission systems. In the section on 'Polarization handling', we give a review for our work on polarization handling, including PBSs and PRs.

Second, optical isolators are very important, but light non-reciprocity is difficult to realize. A well-known approach for optical isolators is using magneto-optic (MO) materials. However, it is difficult to grow MO material on silicon due to the lattice mismatch. In order to solve this problem, we developed a bonding technology to combine MO material with silicon PICs so that an on-chip isolator is realized. ${ }^{20}$

Third, for LS-PICs, low-loss optical waveguides are vital. In particular, rotational velocity sensors, ${ }^{21}$ optical buffers, ${ }^{22}$ true-time-delay beam-steering networks ${ }^{23}$ and other applications requiring long propagation distances and high-Q resonators can benefit from ultra-low propagation loss. According to published results, the propagation loss is usually at the order of $2-3 \mathrm{~dB} \mathrm{~cm}^{-1}$ for silicon strip nanowires, ${ }^{9}$ and $0.27 \mathrm{~dB} \mathrm{~cm}^{-1}$ for shallow-ridge silicon waveguides ${ }^{24}$ fabricated with standard fabrication processes. When an etchless process is used, the loss of SOI nanowires could be as low as $0.3 \mathrm{~dB} \mathrm{~cm}^{-1}$, because the roughness is very small. ${ }^{25}$ However, it is still not low enough for a long propagation distance (e.g., the several-meter-long delay line for an on-chip optical buffer, as shown in Figure 1). ${ }^{14}$ In addition, silicon is not transparent in the wavelength regime of $\lambda<1000 \mathrm{~nm}$, which is important for some applications such as biosensing due to the low optical absorption in water and the transparency of proteins in this wavelength range. ${ }^{26}$ Therefore, an ultra-low loss optical waveguides system is desired for a broad wavelength range from visible to infrared light. Recently, we have developed a novel SiN-on-Si optical waveguide, which shows a world-recorded low loss (i.e., 0.045 $0.04 \mathrm{~dB} \mathrm{~m}^{-1}$ ).

This paper is organized as follows. Polarization handling devices, including PBSs and PRs are covered in the section on 'Polarization handling'. The section on 'Light non-reciprocity' describes optical isolators. In the section on 'Loss reduction', we review recent work on low-loss optical waveguides as well as components (e.g., ring resonators, AWGs and polarizers) made with this technology.

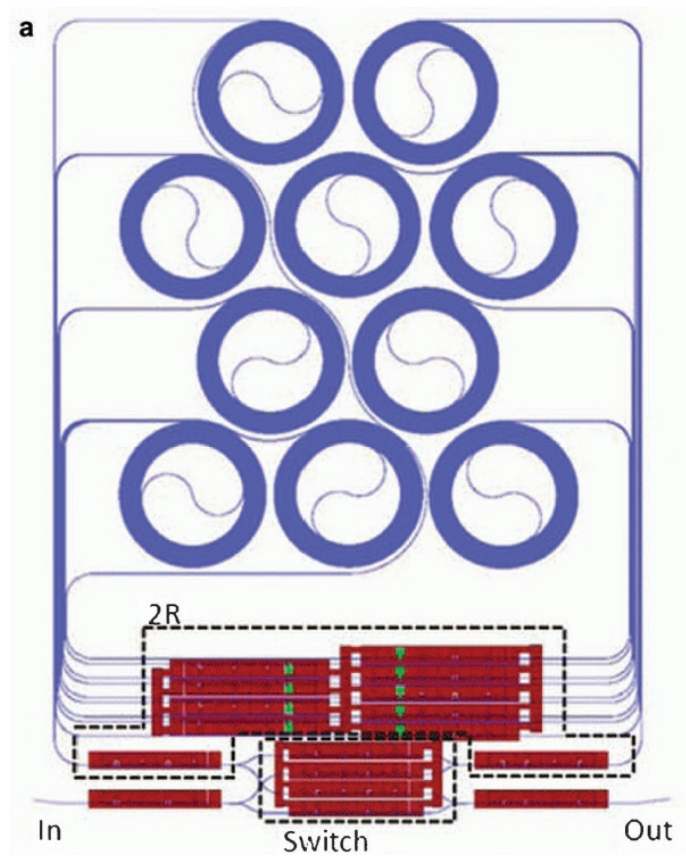

\section{FUNDAMENTAL TECHNOLOGIES FOR FUTURE LS-PICS ON SILICON}

\section{Polarization handling}

On-chip polarization handling devices including polarizers, PBSs and PRs, are important for future LS-PICs. ${ }^{27-30}$ Furthermore, miniature devices are desired, because more and more components are integrated in a single chip as indicated by Moore's law for optical integration. In this section, we review our recent work on ultrasmall silicon polarization-handling devices.

PBS. Several kinds of waveguide type PBSs have been reported by using various structures, e.g., Mach-Zehnder interferometers (MZIs), ${ }^{30,31}$ multimode interference (MMI) structure, ${ }^{32-35}$ directional couplers (DCs) ${ }^{36-41}$ and photonic crystal (PhC)/grating structures. ${ }^{42,43}$

For the conventional MMI-based PBS, the MMI length is usually chosen as the common multiple of the beat lengths for transverse electric (TE) and transverse magnetic (TM) polarizations. A significant difference between the beat lengths of TE and TM polarizations is then needed for a short PBS. However, MMI coupler is usually polarization-insensitive. It makes the PBS quite long, even when SOI nanowires are used. ${ }^{34}$ The MMI-based PBS could be shortened by using quasi-state imaging effect ${ }^{32}$ or by cascading structures. ${ }^{33}$ However, this PBS is still quite long $(\sim 1805 \mu \mathrm{m}$ (Ref. 32$)$ and $\sim 10^{4} \mu \mathrm{m}$ (Ref. 33)).

As a very simple and versatile element, an MZI has been used very widely for optical modulators, optical sensor, etc. By using the birefringence of the MZI arm waveguides, an MZI could be also used to realize polarization splitting. ${ }^{41}$ In order to enhance the birefringence, one usually introduces additional stresses ${ }^{44}$ or uses some highly birefringent materials like $\mathrm{LiNbO}_{3},{ }^{45}$ III-V semiconductor compounds ${ }^{46}$ or liquid crystals. ${ }^{47}$ However, the birefringence is still small and the fabrication is not compatible to the silicon process.

Another possible way to make a short PBS is utilizing the strong polarization dependence of $\mathrm{PhC}$ structures. ${ }^{42,43}$ However, the design is

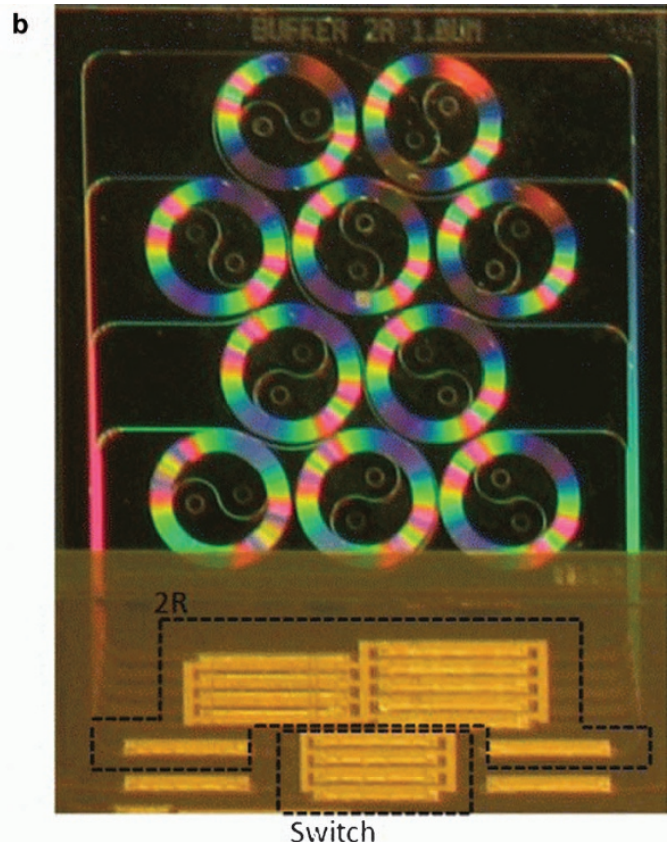

Sivitch

Figure 1 (a) Device layout of the optical buffer; (b) the fabricated device. ${ }^{14}$ 
relatively complex and the fabrication is relatively difficult. More importantly, the PhC structure usually introduces a large loss.

As an alternative, a DC is a popular structure for PBS, because of its simplicity and easy design. A DC-PBS usually consists of two identical coupling waveguides and the length for the coupling region is chosen to satisfy $L=p L_{\pi_{-} \mathrm{TE}}=q L_{\pi_{-} \mathrm{TM}}$, where $L_{\pi_{-} \mathrm{TE}}$ and $L_{\pi_{-} \mathrm{TM}}$ are the coupling lengths for TE and TM polarizations, respectively, $p, q$ are integers, and $q=p+m(m= \pm 1,3,5, \ldots)$. This introduces some limits for the design flexibility and also makes the DC-PBS quite long (e.g., $\sim 1600 \mu \mathrm{m}),{ }^{40}$ especially when one uses low index-contrast $(\Delta)$ waveguides due to the weak birefringence. Furthermore, such a DC-PBS behaves like a series-cascaded DC filter, which definitely has a smaller wavelengthbandwidth than a single-stage DC.

A better design for compact, broad-band and fabrication-tolerant PBSs is to utilize a particular coupling system, in which only one polarization has a strong cross-coupling, while the other polarization is almost not coupled. In this case, the length of the coupling region becomes as short as $L=L_{\pi_{-} \mathrm{TE}}$, or $L=L_{\pi_{-} \mathrm{TM}}$. There are two ways to realize such a particular coupling system. One is using a very strongly polarization-dependent coupling system, in which one has $L_{\pi \_\mathrm{TE}} \gg L_{\pi \_\mathrm{TM}}$ or $L_{\pi_{-} \mathrm{TM}} \gg L_{\pi_{-} \mathrm{TE}}$. In this way, a SOI-nanowire PBS as small as $7 \times 16 \mu \mathrm{m}^{2}$ is realized in Ref. 28 by utilizing $L_{\pi_{-} \mathrm{TE}} \gg L_{\pi_{-} \mathrm{TM}}$. Regarding that TE polarization still has slight evanescent coupling, which prevents to achieve a high extinction ratio, a cascaded structure is needed to achieve higher extinction ratio, which makes the PBS longer and also reduces the bandwidth.

In contrast, an asymmetrical coupling system with a strong birefringence is a potentially better approach for ultra-short PBSs. In such a coupling system, the waveguide dimension is optimized so that the phase matching condition is satisfied to have a complete crosscoupling for only one polarization. For the other polarization, the phase matching condition is not satisfied due to the strong birefringence, and there is almost no cross-coupling. This way, the two orthogonal polarizations are separated within a short length.

The recently developed Si nanowires ${ }^{48}$ and nanoslot waveguides ${ }^{49}$ are very attractive for PBSs, because they do provide a huge birefringence. Symmetric SOI nanowire couplers have been reported for small PBSs. ${ }^{34,35,38,41}$ However, there is not much work on asymmetrical couplers for polarization splitting. In Ref. 29, a PBS with a $16-\mu \mathrm{m}$ long coupling region including two parallel Si nanowires and a nanoslot waveguide in between is presented. The PBS length cannot be reduced further, since the gap between waveguides is necessarily larger than $230 \mathrm{~nm}$ as mentioned in Ref. 29. In the following part, our two kinds of ultra-short PBSs based on asymmetrical couplers are reviewed.

PBS based on an asymmetrical coupler consisting of a SOI nanowire and a nanoslot waveguide ${ }^{50}$. Figure 2 shows the PBS based on an asymmetrical coupling system which includes a SOI nanowire and a nanoslot waveguide. In order to be convenient to integrate with other components in the same chip, the mode converter (similar to that in Ref. 52) between the nanoslot waveguide and the strip nanowire is merged into $S$ bends, which also helps to reduce the footprint of the PBS further. In the coupling region, these two waveguides are designed to satisfy the phase-matching condition for TM polarization to have a complete cross-coupling when choosing the length of the coupling region appropriately. For TE polarized light, however, the phase matching condition is not satisfied and consequently it goes through the SOI nanowire without coupling. Finally, TE- and TM-polarized light are separated within a very short length.

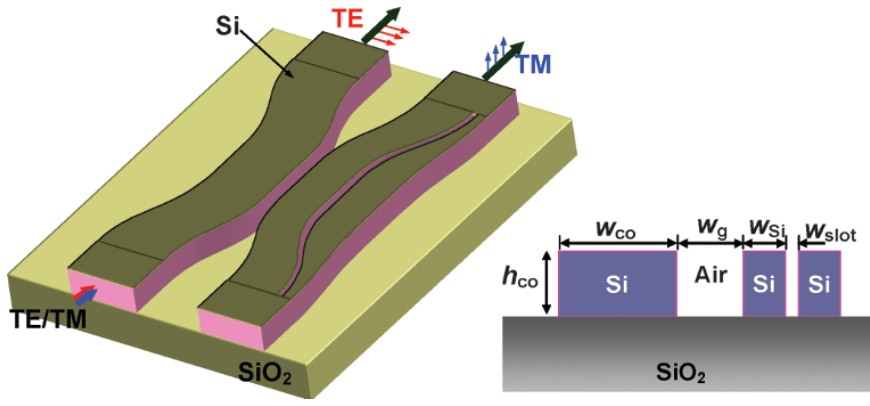

Figure 2 The schematic configuration of the proposed PBS. The inset: the cross-section of the waveguides in the coupling region. PBS, polarization beam splitter; TE, transverse electric; TM, transverse magnetic.

Figure 3 shows the calculated effective indices of a strip nanowire and a nanoslot waveguide as the waveguide core width varies. The nanoslot width is chosen as $w_{\text {slot }}=60,80$ and $100 \mathrm{~nm}$. It can be seen that the effective indices of the TM polarization mode for the two different type waveguides are quite close, and consequently, the phase matching condition can be satisfied (i.e., $n_{\mathrm{TM} 1}=n_{\mathrm{TM} 2}$ ) by choosing the core width $w_{\mathrm{co}}$ and $w_{\mathrm{Si}}$ appropriately (e.g., $w_{\mathrm{co}}=0.4 \mu \mathrm{m}, w_{\mathrm{Si}}=0.26 \mu \mathrm{m}$ and $w_{\text {slot }}=60 \mathrm{~nm}$ ). Then TM-polarized light is cross-coupled completely when choosing the length of the coupling region appropriately (see Figure 4a). On the other hand, TE polarized light has a serious phase mismatch, which depresses the evanescent coupling very significantly, and consequently, it is output from the through port, as shown in Figure $\mathbf{4 b}$.

This design was independently demonstrated in Ref. 53 on a similar PBS. The fabricated PBS has a coupling length of only $13.6 \mu \mathrm{m}$, and the average polarization extinction ratios are about 21 and $17 \mathrm{~dB}$ over the entire C-band for the TE and TM polarizations, respectively.

PBS based on a bent DC $C^{51}$. Figure 5 show the ultra-short PBS consisting of a bent coupling section and an S-bend section with a sharp bend $\left(R_{0}\right)$. The $\mathrm{S}$-bend section is not only for decoupling the two bent waveguides, but also for playing a role as a polarizer, because of the polarization-dependent bending loss in the sharp bent section. This compact polarizer helps to achieve a high extinction ratio in a broad band, as well as a large fabrication tolerance.

The core widths $\left(w_{1}\right.$ and $\left.w_{2}\right)$ for the two bent waveguides in the bent-coupling section are chosen optimally to satisfy the

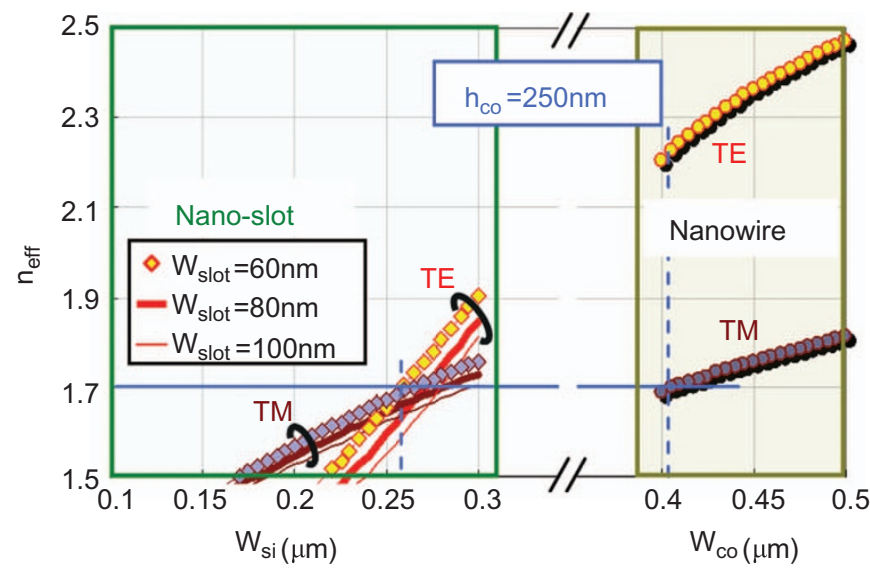

Figure 3 The effective indices of SOI nanowires and nanoslot waveguides. SOI, silicon on insulator. TE, transverse electric; TM, transverse magnetic. 

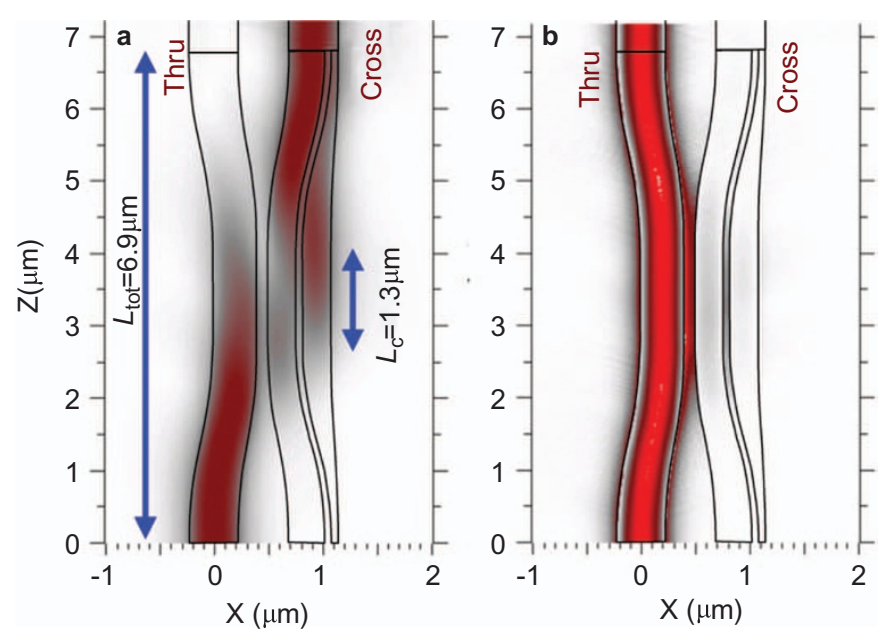

Figure 4 Light propagation in the designed PBS with $w_{\mathrm{co}}=0.4 \mu \mathrm{m}, w_{\mathrm{Si}}=$ $0.26 \mu \mathrm{m}, h_{\mathrm{co}}=250 \mathrm{~nm}, w_{\text {slot }}=60 \mathrm{~nm}$ and $w_{\text {gap }}=100 \mathrm{~nm}$. (a) TM; (b) TE. PBS, polarization beam splitter; TE, transverse electric; TM, transverse magnetic.

phase-matching condition for one polarization (e.g., TM) to have a complete cross-coupling. ${ }^{54}$ For the other polarization (e.g., TE), the phase-matching condition is not satisfied due to the strong waveguide birefringence and it goes through without any significant coupling.

Figure $\mathbf{6 a}$ and $\mathbf{b}$ respectively shows the calculated optical path lengths for both polarization modes as the waveguide width varies. In this example, we choose $R_{2}=20 \mu \mathrm{m}$ and $\Delta R=R_{2}-R_{1}=0.7 \mu \mathrm{m}$ to have a gap width around $200 \mathrm{~nm}$ regarding a single-mode SOI nanowire $\left(w_{\mathrm{co}} \approx 500 \mathrm{~nm}\right)$. The dashed line in Figure 6a gives the optimal widths $\left(w_{1}, w_{2}\right)$ to be phase-matched for TM polarization, e.g., $\left(w_{1}, w_{2}\right)=(0.534 \mu \mathrm{m}, 0.46 \mu \mathrm{m})$. Correspondingly, there is

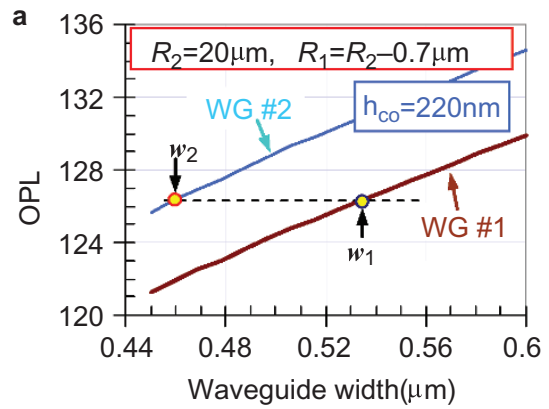

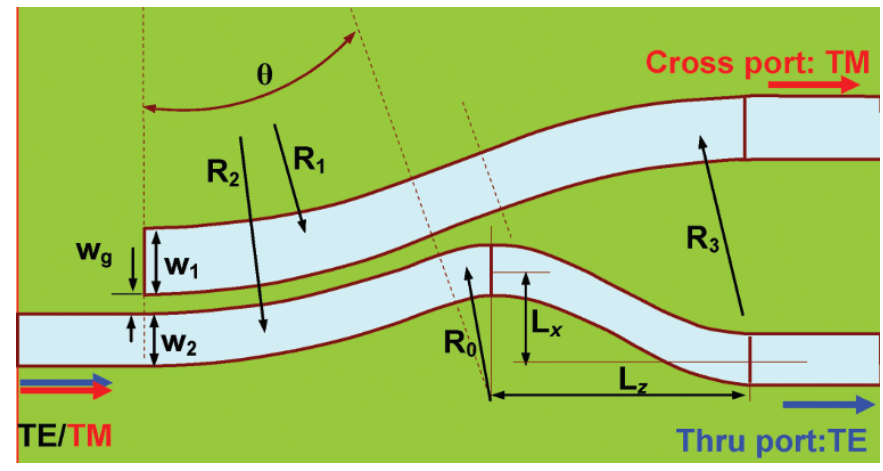

Figure 5 The schematic configuration of the proposed PBS based on a bent DC. DC, directional coupler; PBS, polarization beam splitter; TE, transverse electric; TM, transverse magnetic.

a significant phase mismatch for TE polarization, as shown in Figure $6 \mathbf{b}$.

The S-bend section is designed so that the bending loss is negligible for TE polarization, and the end separation of the two output ports is large enough $(\sim 2 \mu \mathrm{m})$ to be decoupled. The S-bend section includes a sharp bent section $\left(R_{0}=3 \mu \mathrm{m}\right)$ to be the TE-passed polarizer, which helps to filter out the residual TM polarization at the through port and consequently improve the extinction ratio.

Figure $7 \mathbf{a}$ and $\mathbf{b}$ shows the light propagation in the designed PBS for $\mathrm{TE}$ and TM polarizations, respectively. It can be seen that the TM polarization is cross-coupled, while the TE polarization is output from the through port without significant coupling. The total length for the PBS is less than $10 \mu \mathrm{m}$, which is one of the smallest PBS reported until now. Numerical analyses also show that the present PBS has a very large bandwidth $(>200 \mathrm{~nm}$ for an extinction ratio of $10 \mathrm{~dB})$ and a large fabrication tolerance $(> \pm 60 \mathrm{~nm})$.

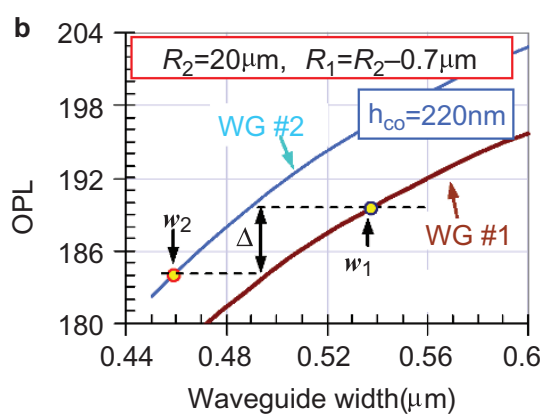

Figure 6 The OPL as the waveguide width varies when $\boldsymbol{R}_{\mathbf{2}}=\mathbf{2 0} \boldsymbol{\mu} \mathbf{m}$. (a) TM; (b) TE. OPL, optical path lengths; TE, transverse electric; TM, transverse magnetic.
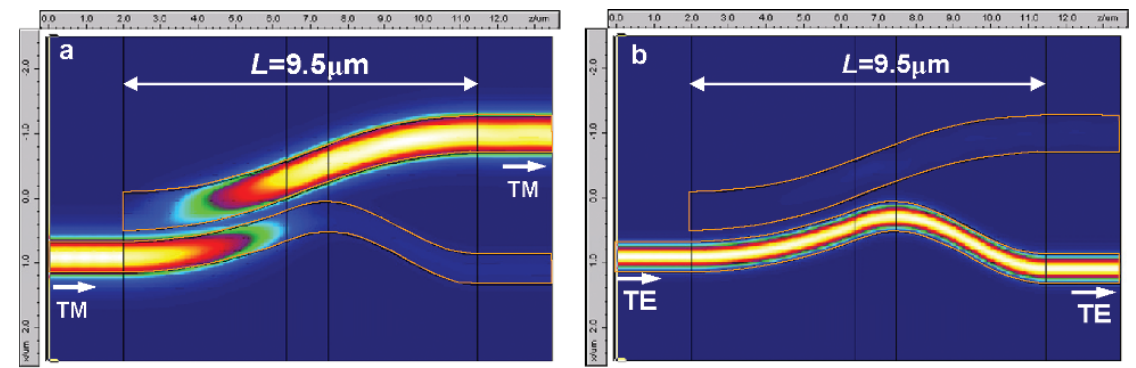

Figure 7 The light propagation in the designed PBS with $L_{\mathrm{dc}}=4.5 \mu \mathrm{m}, R_{1}=19.3 \mu \mathrm{m}, R_{2}=20.0 \mu \mathrm{m}, w_{1}=0.534 \mu \mathrm{m}, w_{2}=0.46 \mu \mathrm{m}$ and $w_{\mathrm{g}}=203 \mathrm{~nm}$. (a) TE; (b) TM. PBS, polarization beam splitter; TE, transverse electric; TM, transverse magnetic. 


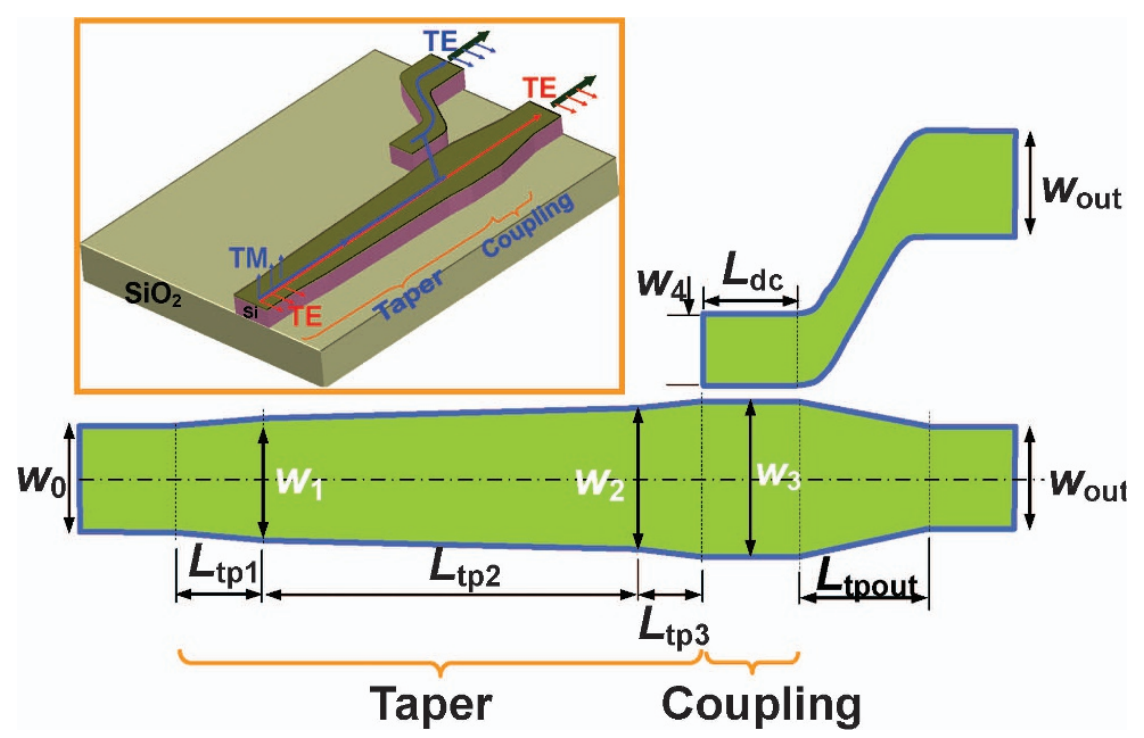

Figure 8 The structure of the proposed PSR. PSR, polarization splitter-rotator; TE, transverse electric; TM, transverse magnetic.

Polarization splitter-rotator (PSR). In comparison with PBSs, it is even more difficult to realize waveguide-type polarization rotators, because a planar waveguide usually has a very good polarizationmaintaining performance and it is not easy to rotate the optical axis of a planar waveguide. In order to do that, one has to introduce some

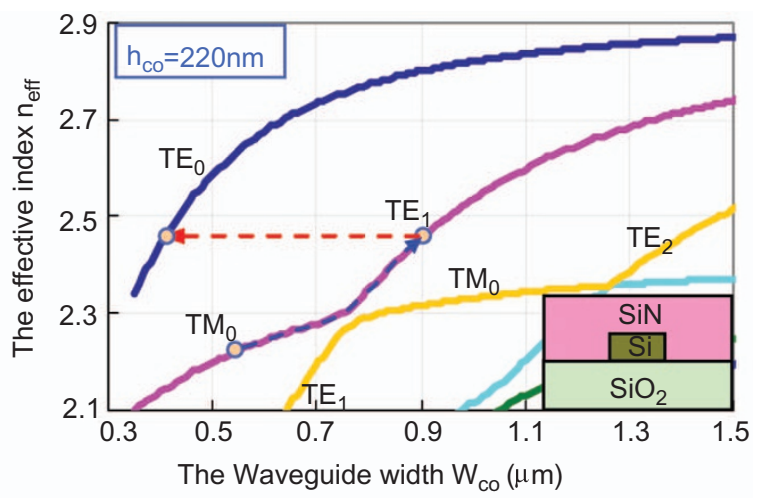

Figure 9 The effective indices for the eigenmodes of SOI nanowires with a $\mathrm{Si}_{3} \mathrm{~N}_{4}$ upper-claddings. Here the thickness of the $\mathrm{Si}$ core layer is $h_{\mathrm{co}}=220 \mathrm{~nm}$. $\mathrm{SOI}$, silicon on insulator; TE, transverse electric; TM, transverse magnetic. specific asymmetrical structures by using off-axis double cores, ${ }^{10}$ cascaded bends, ${ }^{55}$ bilevel tapers, ${ }^{56,57}$ stacked waveguides, ${ }^{58}$ slanted cores, ${ }^{59,60}$ or cores with a cut corner. ${ }^{61}$ These specific structures usually accompany complex and difficult fabrication, e.g., the double etching process with a critical requirement for alignment, or the etching process to achieve a specific angle for the slanted sidewall.

In Ref. 62, a novel concept is proposed for realizing polarization splitting and rotation simultaneously. The total length can be shorter than $100 \mu \mathrm{m}$ and the fabrication is simple, because only one-step etching is needed. Such a PSR consists of a taper and an asymmetrical DC (see Figure 8). In a taper section, the TM fundamental mode might be converted to the higher-order TE mode, due to the mode hybridization in a high index-contrast optical waveguide with an asymmetrical cross-section. As an example, Figure 9 shows the effective indices for all the eigenmodes in a SOI nanowire with an uppercladding of $\mathrm{Si}_{3} \mathrm{~N}_{4}\left(n_{\mathrm{cl}}=2.0\right)$. The dashed curves in Figure 9 show the mode conversions between the $\mathrm{TM}_{0}$ mode and the $\mathrm{TE}_{1}$ mode, when the core width varies. Such a mode conversion is harmful when a lowloss adiabatic taper structure is expected, ${ }^{63}$ while it can be utilized to achieve a PSR as proposed in Ref. 62.

Figure 10a and $\mathbf{b}$ shows the light propagation along the designed PSR. It can be seen that the launched TM polarization is converted to TE polarization and is output from the cross-port, while the launched
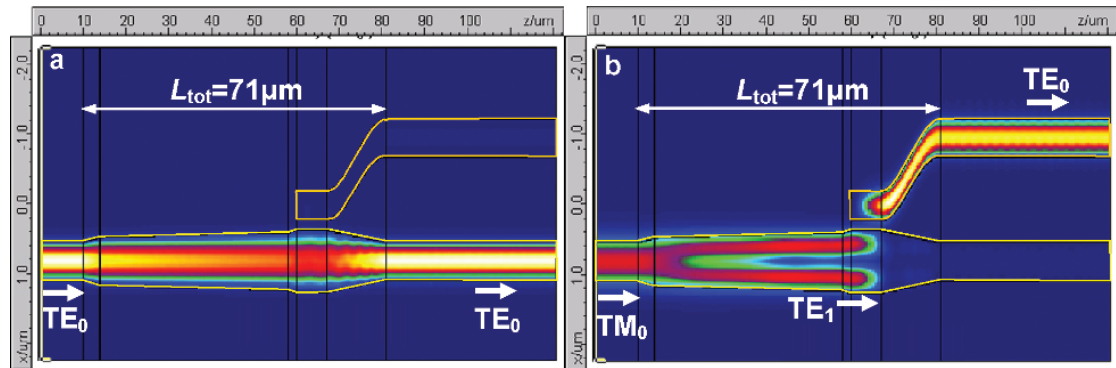

Figure 10 The light propagation in the designed PSR when the input field is TE polarization (a), and TM polarization (b). Here the taper lengths are: $L_{\mathrm{tp} 1}=4 \mu \mathrm{m}$ $L_{\mathrm{tp} 2}=44 \mu \mathrm{m}$ and $L_{\mathrm{tp} 3}=L_{\mathrm{tp} 1}\left(w_{3}-w_{2}\right) /\left(w_{1}-w_{0}\right)$. The other parameters are $w_{0}=0.54 \mu \mathrm{m}, w_{1}=0.69 \mu \mathrm{m}, w_{2}=0.83 \mu \mathrm{m}, w_{3}=0.9 \mu \mathrm{m}, w_{\mathrm{gap}}=0.15 \mu \mathrm{m}$ and $L_{\mathrm{dc}}=7.0 \mu \mathrm{m}$. PSR, polarization splitter-rotator; TE, transverse electric; TM, transverse magnetic. 

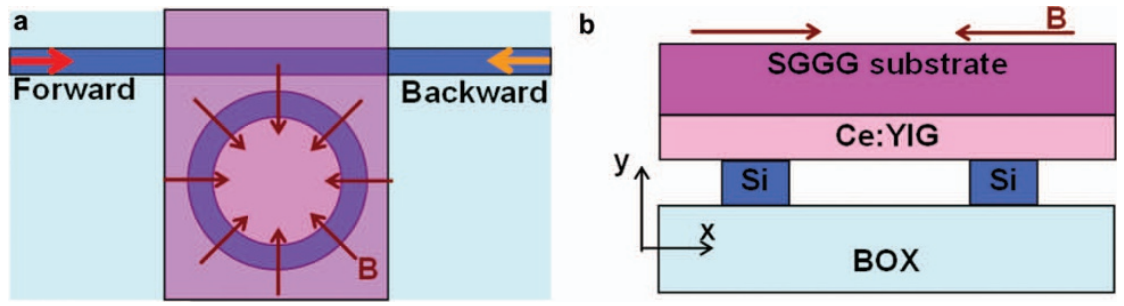

Figure 11 A ring-based optical isolator: (a) top view; (b) cross-section. Ce:YIG, cerium-substituted yttrium iron garnet; SGGG, (Ca, Mg, Zr)-substituted gadolinium gallium garnet.

TE polarization is output from the through port with the same polarization, as expected. The total PSR length is about $71 \mu \mathrm{m}$. Our simulation shows that the designed PSR has a relatively large bandwidth, which is more than $70 \mathrm{~nm}$ for an extinction ratio of $10 \mathrm{~dB}$ and the tolerance for the width deviation is about $-10 \mathrm{~nm}<\Delta w<20 \mathrm{~nm}$ for an extinction ratio of $10 \mathrm{~dB}$. When choosing a relatively long taper (e.g., $L_{\mathrm{tp} 1}>10 \mu \mathrm{m}$ ), a larger bandwidth and fabrication tolerance is expected.

\section{Light non-reciprocity ${ }^{20}$}

Optical isolators and optical circulators are two typical non-reciprocal optical devices. The most important aspect for realizing isolators and circulators is to break the reciprocity of light. For this purpose, Faraday rotators based on MO materials (e.g., iron, cobalt and yttrium iron garnet (YIG)) are often used together with a pair of polarizers. However, the bulky elements make it difficult to be integrated.

Recently, several integrated-type isolators have been explored by using nonreciprocal effects, like mode conversion, ${ }^{64}$ waveguide loss, ${ }^{65}$ phase shift. ${ }^{66,67}$ These approaches have some disadvantages, e.g., difficulty to have phase matching between modes, ${ }^{64}$ high power consumption. ${ }^{65}$ In Refs. 68 and 69, the MZI optical isolators have been demonstrated with the help of nonreciprocal phase shift. A ring resonator is another popular structure for optical isolators, because of the footprint miniaturization. ${ }^{70}$ For example, in Refs. 71 and 72, a ring optical isolator was demonstrated by using a nonlinear effect, which, however, needs specific operation power. More importantly, it does not work when forward and backward light is launched simultaneously.

A ring-based optical isolator on silicon with bonded MO garnets is realized for the first time, as shown in Figure $11 .{ }^{20}$ Cerium-substituted
YIG (Ce:YIG) is chosen as the MO material, because of its high Faraday coefficient and low optical loss $\left(10-15 \mathrm{~dB} \mathrm{~cm}^{-1}\right)$ in the 1550 -nm regime. When a radial magnetic field centered at the ring resonator is applied (see Figure 11), the clockwise- and counterclockwise-propagating modes have different propagation constants and thus different resonance wavelengths. A maximal nonreciprocal effect is obtained when choosing 220-nm-thick silicon cores. However, on the other hand, the mode loss also becomes significant, due to the loss in the MO material. To have a reasonable nonreciprocal effect and low optical loss, the Si core thickness is chosen as $295 \mathrm{~nm}$. A 500-nm-thick Ce:YIG thin film is grown on a $(\mathrm{Ca}, \mathrm{Mg}, \mathrm{Zr})$-substituted gadolinium gallium garnet substrate as the MO material used here and the Faraday rotation coefficient of Ce:YIG is about $-4500^{\circ} \mathrm{cm}^{-1}$ at the $1550-\mathrm{nm}$ wavelength regime. ${ }^{73}$ The $\mathrm{O}_{2}$ plasma-assisted low-temperature bonding technology ${ }^{69}$ is used to bond the MO material on the fabricated silicon rings. One-hour annealing at $250{ }^{\circ} \mathrm{C}$ is followed to have a strong bond.

The fabricated optical isolator is characterized by measuring the transmission at the through port. TM-polarized light is input through a lensed fiber. Figure 12a shows the transmission spectra of the ring isolator with external magnetic fields in different directions, which are equivalent to the situation with different optical propagation directions with a fixed magnetic field. The shift of the resonance wavelength due to the nonreciprocal effect is observed as expected. This wavelength shift is proportional to the magnitude of the magnetic field until the Ce:YIG saturates. For the present case, an isolation ratio of about $9 \mathrm{~dB}$ is achieved when the resonance wavelength shift is equal to half of the free spectral range (FSR). The limit isolation is mainly due to the higher cavity loss and the lower extinction ratio when the ring has an MO material on the top. This can be seen from Figure 12b,
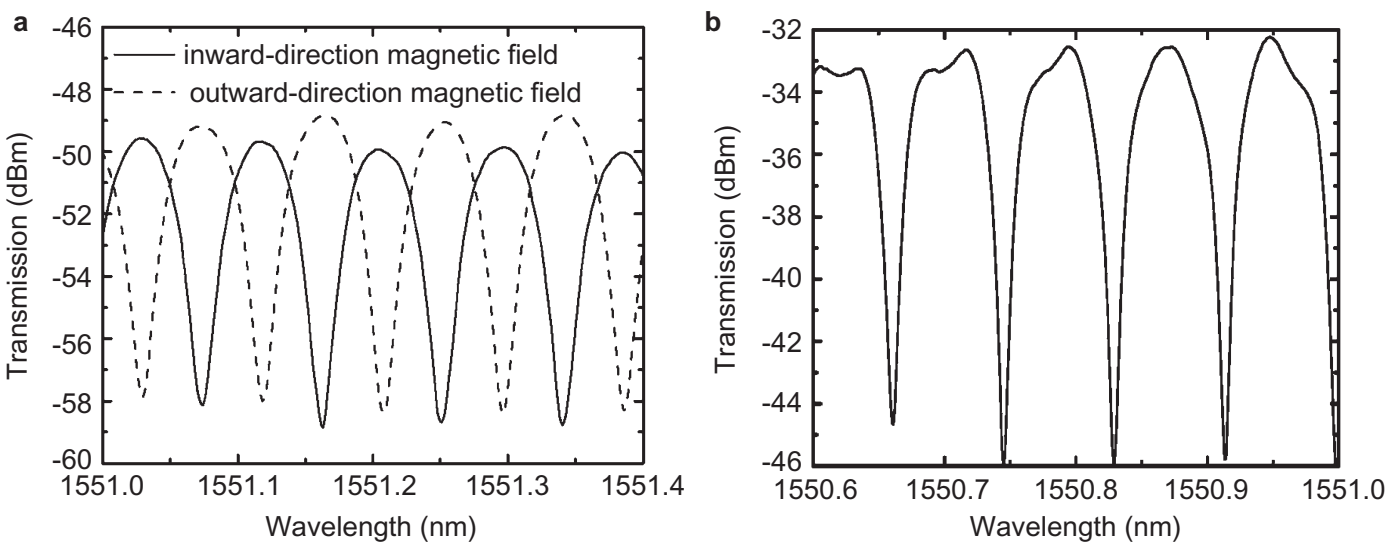

Figure 12 (a) Transmission spectra of the ring isolator with external radial magnetic fields in different directions. (b) Transmission spectrum of the ring resonator before bonding with Ce:YIG. Ce:YIG, cerium-substituted yttrium iron garnet. 
which shows the transmission spectrum of the ring resonator before bonding with Ce:YIG for comparison. In order to improve the isolation ratio, the ring resonator should be modified to have a critical coupling for a higher extinction ratio. The isolation can be improved further by cascading structures. Besides, it is also essential to reduce the ring loss for lower isolation loss and higher isolation. One has to further optimize fabrication processes to have a high-quality etching for silicon nanowaveguides, as well as bonding process. Finally, lower MO material loss will definitely help improve the performance of the isolator. A smaller bending radius for the ring is also desired to have a smaller footprint, as well as a larger FSR. ${ }^{74}$ Such a Si platform with bonded MO material is also useful to realize more complicated integrated non-reciprocal elements (e.g., circulators) and it is promising for the future LS-PICs, since the fabrication process is CMOScompatible.

\section{Loss reduction}

SOI nanowires usually have a scattering loss on the order of $0.3-3 \mathrm{~dB}$ $\mathrm{cm}^{-1,9,24,25}$ and do not work in the wavelength range of $\lambda<1000 \mathrm{~nm}$. Therefore, some alternatives for low-loss optical waveguides on silicon are still desired. One approach is to deposit low index-contrast (often $<1 \%$ ) silica cores. ${ }^{75,76}$ The lowest-loss waveguides at $\lambda=1550 \mathrm{~nm}$ are the single-mode phosphorus-doped waveguides ${ }^{75}$ and the 'quasisingle-mode' Ge-doped waveguides ${ }^{76}$ with $0.85 \pm 0.03 \mathrm{~dB} \mathrm{~m}^{-1}$ and $0.3 \mathrm{~dB} \mathrm{~m}^{-1}$ propagation losses, respectively. However, the low index-contrast limits its flexibility for compact applications.

In our recent work, the $\mathrm{Si}_{3} \mathrm{~N}_{4}$-on- $\mathrm{SiO}_{2}$ system is considered, because $\mathrm{Si}_{3} \mathrm{~N}_{4}$ and $\mathrm{SiO}_{2}$ have a transparent window from visible light to infrared light, and the stoichiometric nature of these two materials makes it easier to get uniform characteristics across large wafer sizes. ${ }^{77}$ This system is very useful for various applications, not only optical communications around 1.2-1.6 $\mu \mathrm{m}$, but also for optical sensing in the visible light range. The $\mathrm{Si}_{3} \mathrm{~N}_{4}$ optical waveguide has a high aspect ratio $\mathrm{Si}_{3} \mathrm{~N}_{4}$ core (see Figure 13) to minimize the scattering loss at the sidewall, which is the dominant loss mechanism. With an optimized fabrication process, the $\mathrm{Si}_{3} \mathrm{~N}_{4}$-on- $\mathrm{SiO}_{2}$ optical waveguide shows a loss as low as $0.045 \pm 0.04 \mathrm{~dB} \mathrm{~m}^{-1}$, which is presently a world record for planar waveguides. Several typical photonic integrated devices, e.g. MMI splitters, AWGs, ${ }^{78}$ ultra-high Q ring resonators ${ }^{79}$ and polarizers, have also been demonstrated.

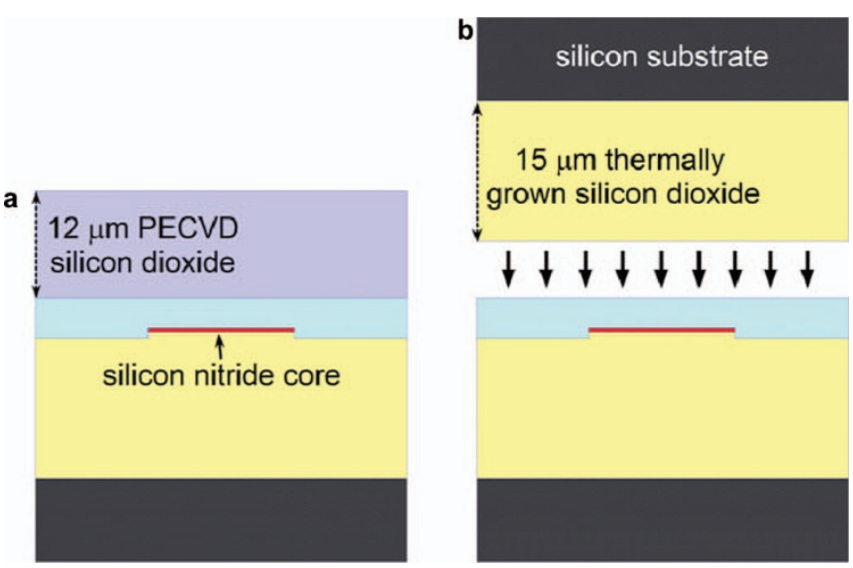

Figure 13 The schematic cross-sections of (a) first and (b) second generation low-loss $\mathrm{Si}_{3} \mathrm{~N}_{4}$ optical waveguides.
Low-loss $\mathrm{Si}_{3} \mathrm{~N}_{4}$ optical waveguide. The optical waveguides are fabricated on $\mathrm{Si}$ substrates with $15-\mu$ m-thick thermally grown $\mathrm{SiO}_{2}$. A very thin $(<100 \mathrm{~nm}) \mathrm{Si}_{3} \mathrm{~N}_{4}(n=1.98)$ film is then deposited using low pressure chemical vapor deposition. The waveguide cores are then defined with a dry etch that extends fully through the $\mathrm{Si}_{3} \mathrm{~N}_{4}$ layer. A 3.1- $\mu$ m-thick $\mathrm{SiO}_{2}$ layer is then deposited in three steps using low pressure chemical vapor deposition. The surface is planarized with a chemical mechanical polishing step. Then a $12-\mu \mathrm{m}$-thick $\mathrm{SiO}_{2}$ uppercladding is deposited with the Plasma-enhanced chemical vapor deposition (PECVD) technology before a 3-h annealing at $1150{ }^{\circ} \mathrm{C}$. In order to reduce the loss further, the deposited PECVD top cladding is replaced with a high-quality thermal oxide by means of $\mathrm{O}_{2}$ plasmaenhanced wafer bonding. The bond is strengthened by a 3-h annealing at $950{ }^{\circ} \mathrm{C}$.

The first-generation low-loss $\mathrm{Si}_{3} \mathrm{~N}_{4}$ optical waveguides with a $\mathrm{SiO}_{2}$ upper-cladding fabricated by the PECVD technology. For the firstgeneration low-loss $\mathrm{Si}_{3} \mathrm{~N}_{4}$ optical waveguide with a PECVD $\mathrm{SiO}_{2}$ upper-cladding, the single-mode propagation loss is as low as $0.70 \pm 0.02 \mathrm{~dB} \mathrm{~m}^{-1}$. It was measured by using a 3.18 -m-long test spiral structure as shown in Figure 14. The minimum bend radius of the center S-bend is $R=10 \mathrm{~mm}$, which is large enough to have a negligible bending loss. The measured loss in these waveguides is limited by the quality of the PECVD deposited layer on top of the $\mathrm{Si}_{3} \mathrm{~N}_{4}$ waveguide. Higher quality claddings are necessary for lower loss waveguides as shown below.

The second-generation low-loss $\mathrm{Si}_{3} \mathrm{~N}_{4}$ optical waveguide with bonded oxide upper-cladding. The second-generation low-loss $\mathrm{Si}_{3} \mathrm{~N}_{4}$ optical waveguide is realized with a bonded high-quality thermal oxide instead of a PECVD oxide for the upper-cladding. Because $15-\mu \mathrm{m}$ thick thermal oxide wafers can be batch-oxidized and consequently be available for bonding immediately, this wafer bonding process is faster and cheaper than the PECVD process (which takes $>20 \mathrm{~h}$ ).

Twelve 1-m-long spiral waveguides with core widths ranging from 3 to $14 \mu \mathrm{m}$ (see the inset in Figure 15) are measured with an optical backscatter meter. Figure 15 shows the critical bend radius for each width, where we define the critical bend radius as the radius at which bend loss equals $0.1 \mathrm{~dB} \mathrm{~m}^{-1}$. The theoretical critical bending radius from simulation with fit refractive indices is also shown by the dashed curves in Figure 15. For the higher confinement waveguides, there is a sharp knee in the backscatter data where the bend loss begins to dominate, giving good agreement between the theoretical and experimental data. For waveguides with lower confinement, the bend loss is not negligible at the starting radius of the spiral structure, and the agreement starts to degrade as the knee in the backscatter data softens. The core/cladding refractive indices of $1.98 / 1.45$ used for the

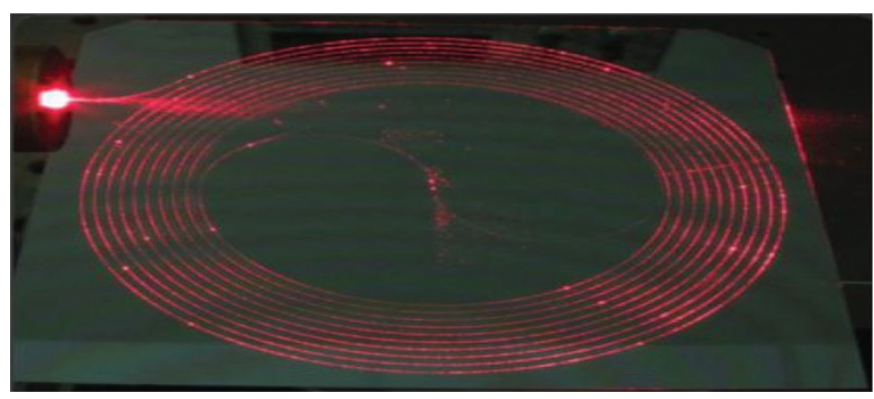

Figure 14 An array of twenty 3.18-m-long spiraled waveguides. 


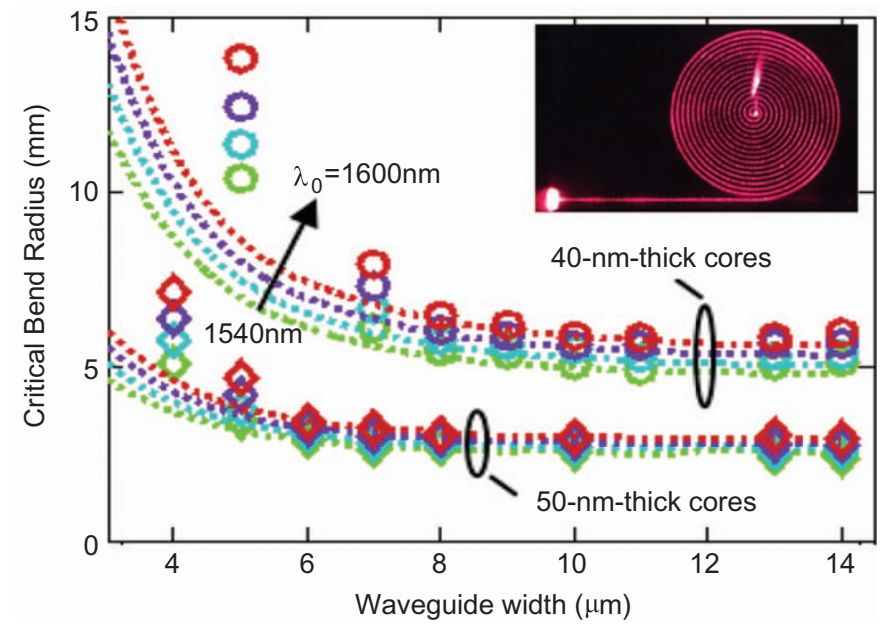

Figure 15 Critical-bend-radius vs. waveguide width at 1540, 1560, 1580 and $1600 \mathrm{~nm}$ wavelengths. Dashed lines are simulated values.

theoretical fits to the data in Figure $\mathbf{1 5}$ are within 0.01 of the measured material values, indicating a minimal change to the refractive index due to the stress-optic effect. In contrast, the first generation $\mathrm{Si}_{3} \mathrm{~N}_{4}$ optical waveguide has residual stress due to the PECVD and higher temperature annealing processes. This residual stress lowers the refractive index contrast so that the substrate leakage and bending losses are higher for a given core geometry. Critical bend radius data for the first generation waveguides give a theoretical fit core index of 1.90 , which is a factor of 8 increase in the index perturbation compared with the second generation waveguides in Figure 15.

Figure 16 shows the propagation loss in two broad wavelength ranges, centered near 1.307 and $1.56 \mu \mathrm{m}$, so that one can see the spectral dependence of the fit molecular absorption and interfacial scattering loss contributions. The absorption loss peaks are fit to Gaussian functions, the center wavelengths of which are given in the figure color keys, while the scattering loss is fit to a function shape obtained from numerical simulation of the spectral dependence of interfacial scattering loss. ${ }^{77}$ The minimum propagation losses measured in the regimes around 1.3 and $1.58 \mu \mathrm{m}$ are $0.33 \pm 0.03 \mathrm{~dB} \mathrm{~m}^{-1}$ and $0.045 \pm 0.04 \mathrm{~dB} \mathrm{~m}^{-1}$, respectively. The spectral flatness in the $1.3 \mu \mathrm{m}$ regime is not observed in first generation waveguides, so the absence of an absorption loss peak in this regime for the wafer-bonded waveguides suggests a mitigation of $\mathrm{OH}$ absorption loss there. It should be possible to reduce the hydrogen impurity concentration in the waveguides with process changes in order to lower the loss further. In comparison with the first generation, the second generation has record low propagation loss of $<0.1 \mathrm{~dB} \mathrm{~m}^{-1}$, shorter fabrication time, mitigated absorption loss and lower dielectric film stress.

Ultra-high-Q ring resonators ${ }^{79}$. Ultra-high-Q optical resonators are crucial to many applications, e.g., nonlinear optics, ${ }^{80}$ biosensing, ${ }^{81}$ telecommunications ${ }^{82}$ and microwave photonic filters. ${ }^{83}$ Whisperinggallery-mode resonators with ultra-high-quality factor $(\mathrm{Q})$ of $10^{8}$ have been demonstrated in microtoroidal structures made from thermally grown silica. ${ }^{84}$ However, their nonplanar structure makes it difficult to integrate with other optical devices for complex functionality. Among various materials available for on-chip resonators, $\mathrm{SiO}_{2}, \mathrm{SOI}$ and $\mathrm{Si}_{3} \mathrm{~N}_{4}$ are good candidates, because of the availability of low-loss planar optical waveguides.

Planar silicon microdisk resonators were reported to have $\mathrm{Q}$ factors of 3 million at $1550 \mathrm{~nm} .{ }^{85}$ However, the resonance is in a high-order mode, and Si is not available for the visible light, which is important for some ring resonator applications like biosensing. ${ }^{26}$ In the $\mathrm{Si}_{3} \mathrm{~N}_{4}$ on- $\mathrm{SiO}_{2}$ platform, people have demonstrated ring resonators with $\mathrm{Q}$ factors up to 3 million at $1550 \mathrm{~nm},{ }^{86}$ and microdisk resonators with Q factors of 3.4 million at $652-660 \mathrm{~nm} .{ }^{87}$ Higher $Q$ factor is expected for $\mathrm{Si}_{3} \mathrm{~N}_{4}$-on-Si resonators to satisfy the demands of many applications.

Recently, we demonstrated planar $\mathrm{Si}_{3} \mathrm{~N}_{4}$ ring resonators with integrated DCs fabricated with our ultra-low-loss high-aspect-ratio waveguide technology. ${ }^{79}$ Regarding the ultra-narrow 3-dB bandwidth,
Loss Contributions:

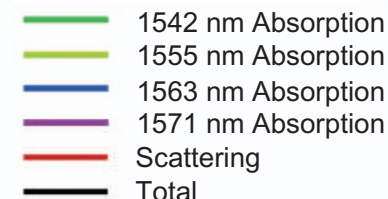

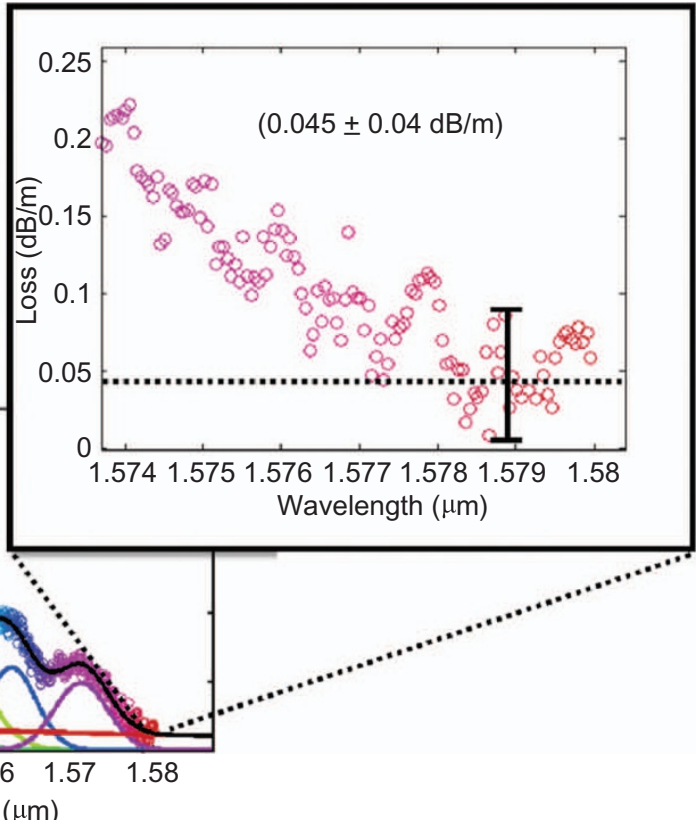

Figure 16 Propagation loss vs. wavelength for a waveguide with a 40 -nm-thick by $13-\mu \mathrm{m}$-wide core and bonded thermal oxide upper cladding. 

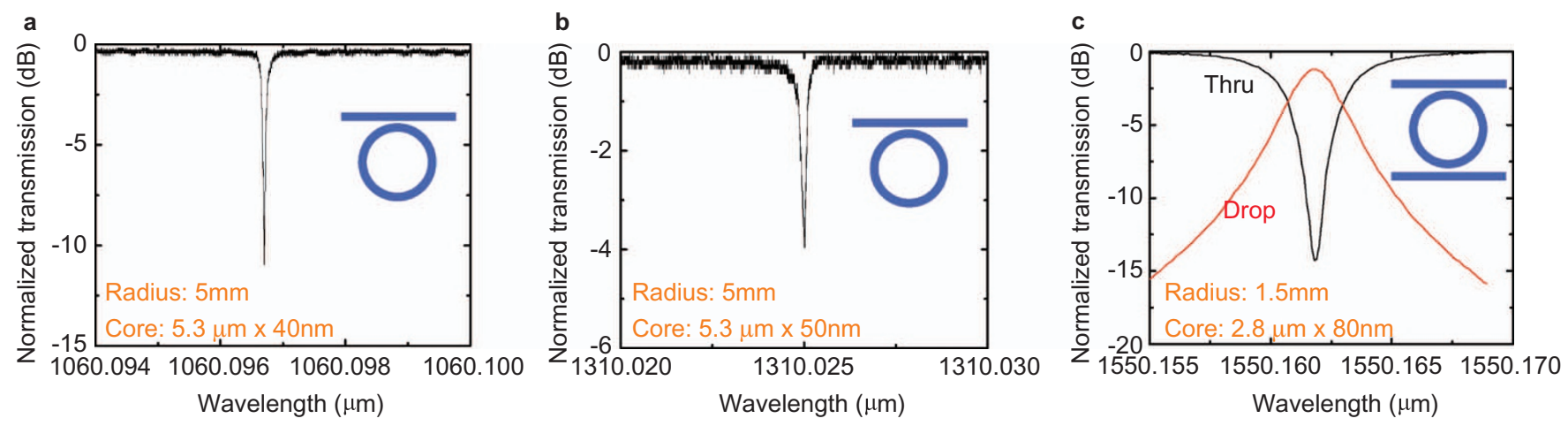

Figure 17 Transmission spectra of ring resonators when operating at (a) $1060 \mathrm{~nm}$, (b) $1310 \mathrm{~nm}$ and (c) $1550 \mathrm{~nm}$.

a narrow-linewidth tunable laser source and a synchronized oscilloscope were used to resolve the transmission spectrum. The input polarization is set at the TE mode, because the ring resonator is designed to have low loss for TE polarization. ${ }^{89}$

Figure 17 shows the transmission spectra of ring resonators operating in the wavelength regimes of 1060, 1310 and $1550 \mathrm{~nm}$, respectively, and the corresponding thicknesses of the $\mathrm{Si}_{3} \mathrm{~N}_{4}$ core are 40,50 and $80 \mathrm{~nm}$. The characterization of the transmission spectra in three different wavelength regimes helps to understand the sources of loss. The highest $\mathrm{Q}$ factor of the resonator operating around $1060 \mathrm{~nm}$ is up to $18-19$ million, which is the highest Q reported for planar ring resonators at $1060 \mathrm{~nm}$. For the resonator operating at $1310 \mathrm{~nm}$, the intrinsic Q factor extracted from the measurement is about 28 million and the corresponding propagation loss is $1.1 \mathrm{~dB} \mathrm{~m}^{-1}$. For the adddrop ring resonator operating at $1550 \mathrm{~nm}$ with a smaller radius and core width, the estimated propagation loss is as low as $2.9 \mathrm{~dB} \mathrm{~m}^{-1},{ }^{77}$ and the intrinsic $\mathrm{Q}$ value is about 7 million. The corresponding 3- $\mathrm{dB}$ bandwidth of the add-drop filter is approximately $300 \mathrm{MHz}$, which can be used as a microwave photonic filter for channel selection.

Low-loss $A W G$ (de)multiplexer ${ }^{78}$. As an important element for wavelength division multiplexed technology, ${ }^{90-93}$ a low-loss $\mathrm{Si}_{3} \mathrm{~N}_{4}$ AWG is demonstrated, as shown in Figure 18a. Because the waveguide has an ultra-thin core layer (e.g., $50 \mathrm{~nm}$ ), it is easy to refill the upper-cladding material into the gaps even when the gap width is reduced to less than $2 \mu \mathrm{m}$. Regarding the resolution limitation of the UV-lithography process, $1-\mu \mathrm{m}$-wide gaps are used to reduce the transition loss. In addition, the ultra-thin core layer allows a very wide single-mode optical waveguide, which is more tolerant to the width variation. Thus, it helps to obtain a low-crosstalk AWG (de)multiplexer.

A measurement setup consisting of a free-space optical system with a bulk polarizer ${ }^{94}$ is used for the measurement of the AWG. Figure 18b shows the measured spectral responses of all the channels in the wavelength range from 1310 to $1335 \mathrm{~nm}$. The FSR of the AWG becomes $18.4 \mathrm{~nm}$ and only 13 channels are available in an FSR. The crosstalk from the adjacent channel is less than $-30 \mathrm{~dB}$ and the non-adjacent channel crosstalk is about $-40 \mathrm{~dB}$. The low crosstalk and the low loss make the AWG attractive for integrated microspectrometers in the future.

Polarizer with a high extinction ratio. As mentioned in the section on 'Polarization handling', a polarizer is one of the basic polarization handling devices. The basic principle of the polarizer is to make one polarization more lossy than the other one. In order to have such polarization dependent loss (leakage or absorption losses), one usually uses an optical waveguide with significant birefringence,
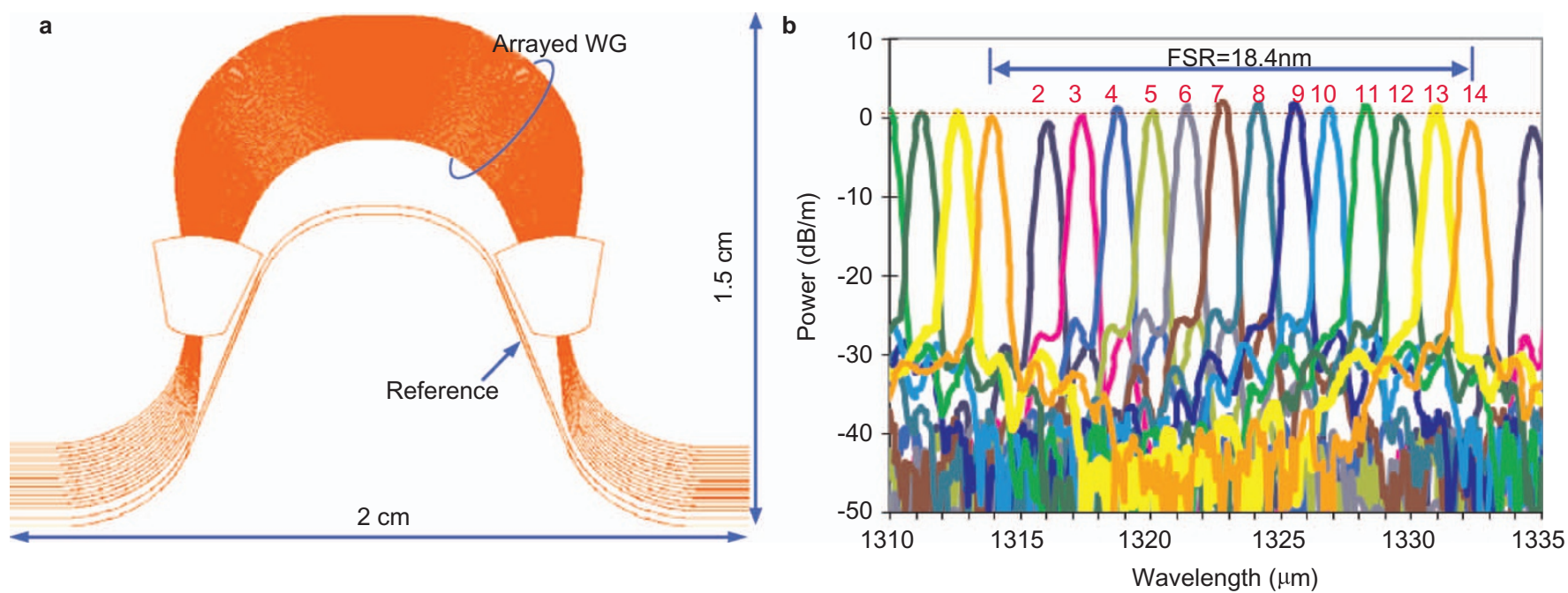

Figure 18 (a) The AWG layout; (b) the measured spectral responses of all the channels in the wavelength range around 1310 nm. AWG, arrayed-waveguide grating; FSR, free spectral range. 

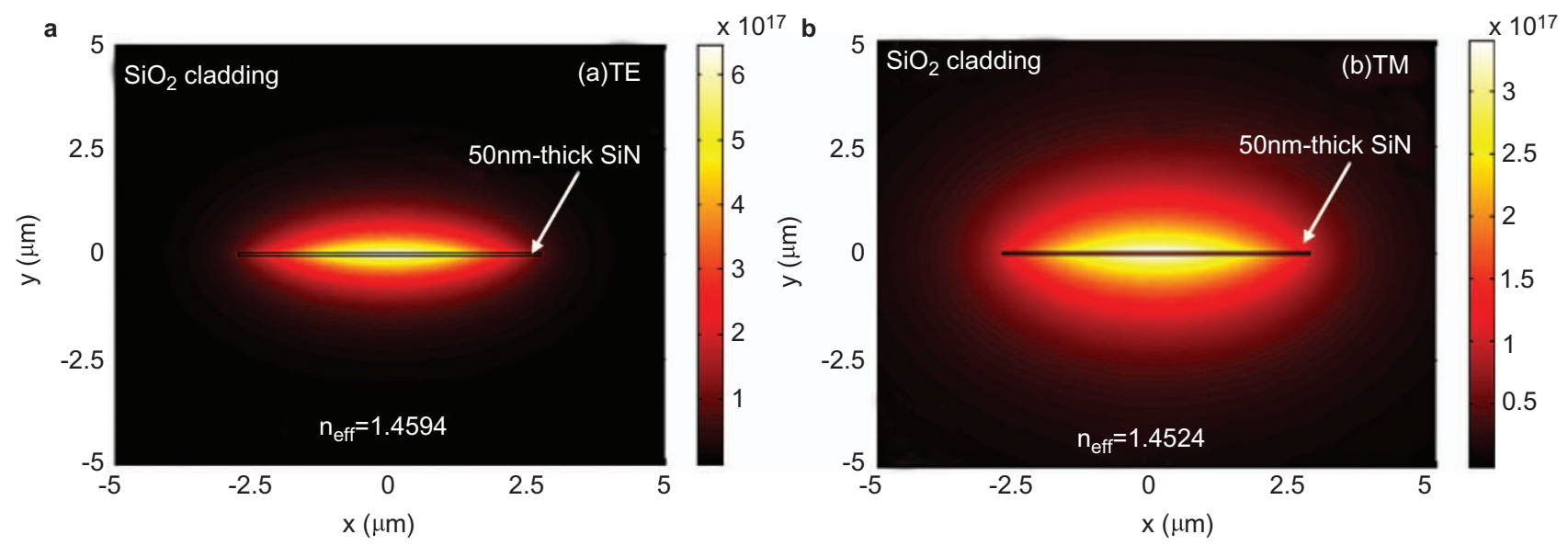

Figure 19 (a) The mode profile of the TE-polarized fundamental mode; (b) the mode profile of the TM-polarized fundamental mode. TE, transverse electric; TM, transverse magnetic.

e.g., an antiresonant reflecting optical waveguide, ${ }^{95}$ a metal-dielectric waveguide, ${ }^{96} \mathrm{a} \mathrm{LiNbO}_{3}$ waveguide, ${ }^{97}$ a $\mathrm{PhC}$ structure, ${ }^{98}$ liquid crystals, ${ }^{99}$ or a silicon nanophotonic waveguide. ${ }^{100}$ In Ref. 94, a pure silicon TE-passed polarizer based on a shallowly-etched straight optical waveguide is proposed and demonstrated.

Note that the $\mathrm{Si}_{3} \mathrm{~N}_{4}$ waveguide presented above has a very high aspect ratio. Consequently, the mode property of such an optical waveguide is expected to be very polarization dependent, as shown in Figure 19a and $\mathbf{b}$. This makes it a good candidate for realizing polarizers. Numerical simulations have shown that the bending loss of the TM polarization is much higher than that of the TE polarization. ${ }^{89}$ Therefore, a simple low-loss TE-pass polarizer can be realized by using a bending structure. For some applications, such as an optical delay line spiral, the polarizing bend is inherent to the structure. For others, a series of S-bends can yield a high extinction of the TM mode.

Figure 20 compares the normalized transmission of the TE and TM polarized modes through straight waveguides and S-bends. The waveguides are fabricated with wafer-bonded thermal oxide upper-cladding, and the cores are $40 \mathrm{~nm}$ thick by 4,5 or $6 \mu \mathrm{m}$ wide. The extinction of the TM mode after propagation through a 21-mm-long straight waveguide, seen in Figure 20a, is due to the greater coupling loss for the TM mode and the larger leakage loss to the Si substrate. The highest extinction, seen at $\lambda=1620 \mathrm{~nm}$ for the $4-\mu \mathrm{m}$-wide core, is $15 \mathrm{~dB}$. The high extinction of the TM mode after propagation through the S-bend structure seen in Figure 20b is primarily due to a large difference in bend loss for the TE and TM modes. The S-bends have $9.8 \mathrm{~mm}$ bend radii, and no offset is used for mode matching at the bend-tobend or straight-to-bend interfaces. The 6 - and 5 - $\mu \mathrm{m}$ core structures are low loss for the TE mode with an average extinction of 36.8 and $39.8 \mathrm{~dB}$ across the wavelength range. The 4 - $\mu \mathrm{m}$-wide core has a larger average extinction of $45.6 \mathrm{~dB}$, but the TE mode has greater bend loss at this core width, adding significant loss to the polarizer at wavelengths larger than $1530 \mathrm{~nm}$. Using cleaved SMF-28 fibers and index matching gel, the TE insertion loss of the 6 - and 5- $\mu \mathrm{m}$-wide core structures is 3.6 $\mathrm{dB}$. With input and output coupling tapers, the total TE insertion loss can be lower than $1.5 \mathrm{~dB}^{88}$
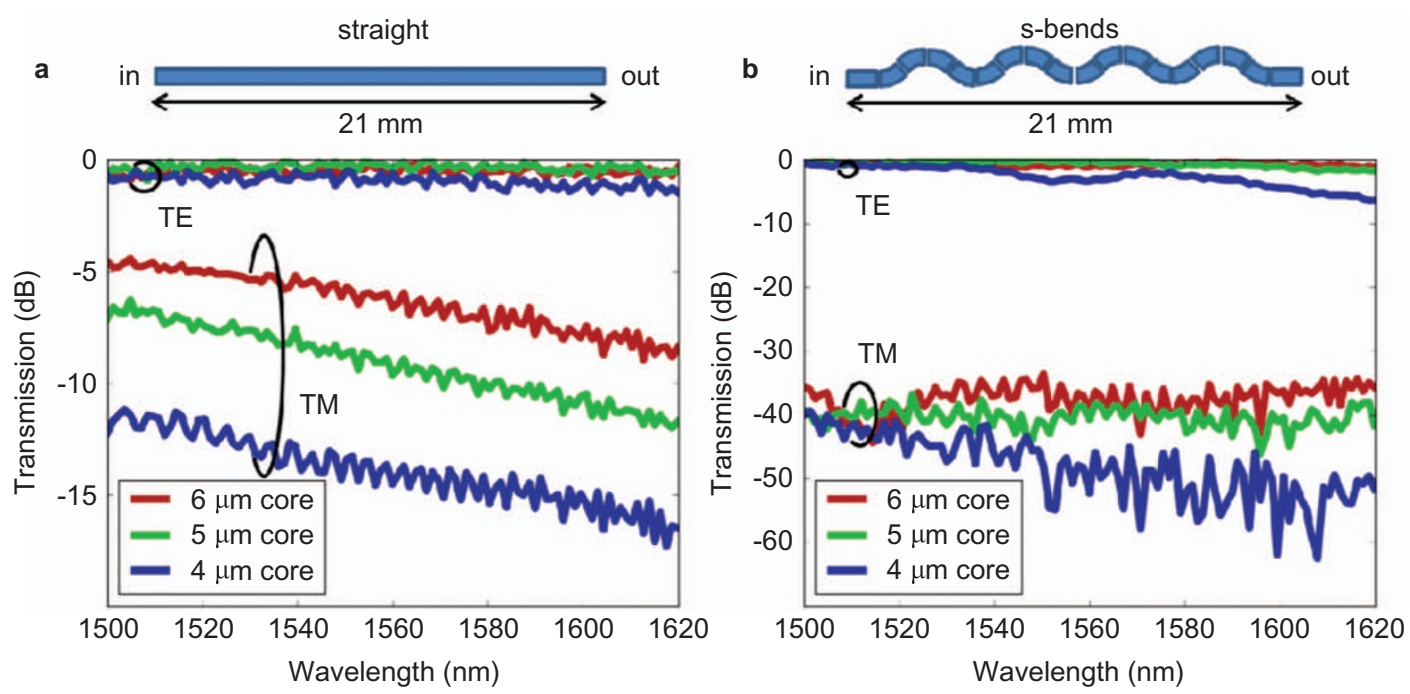

Figure 20 (a) TE and TM transmission through straight 21-mm-long waveguides with a 40-nm-thick core; (b) TE and TM transmission for the same core geometries through a series of $16 \mathrm{~S}$-bends (eight shown above). TE, transverse electric; TM, transverse magnetic. 


\section{CONCLUSIONS}

In this paper, we have given a review of recent work on several fundamental passive technologies for silicon-based LS-PICs, including polarization handling, optical isolators and waveguide loss reduction, which are very important for various applications like optical transmitters, receivers and optical buffers. Polarization handling devices, which include PBSs and polarization rotators, are very important not only for polarization-multiplexing system, but also for polarizationtransparent PICs. We have reviewed our novel designs for ultra-short PBSs by using asymmetrical DCs, as well as our novel concept for realizing a PSR. Recent work on silicon-based ring optical isolators, which is a basic element for a light system (e.g., laser) to avoid any undesired reflections, has been also reviewed. The ring optical isolator is with the help of MO material by bonding technology. Finally, since low-loss waveguides are another fundamental technology for PICs, we have presented our ultra-low-loss optical waveguide by designing a $\mathrm{Si}_{3} \mathrm{~N}_{4}$ core with a very high aspect ratio (the width $\gg$ the height). The loss is reduced to be less than $0.1 \mathrm{~dB} \mathrm{~m}^{-1}$ with an improved fabrication processes by replacing the regular PECVD-deposited $\mathrm{SiO}_{2}$ upper-cladding with a high-quality thermal oxide by means of wafer bonding. With our novel ultra-low-loss $\mathrm{Si}_{3} \mathrm{~N}_{4}$ optical waveguides, we have also realized ultra-high-Q ring resonators, ultra-low loss 16-channel AWG (de)multiplexers, and simple polarizer with high extinction ratio.

\section{ACKNOWLEDGMENTS}

This research is supported by DARPA MTO under the CIPhER contract no. HR0011-10-1-0079 and iPHOD contract no. HR0011-09-C-0123. The authors thank S Rodgers, D Blumenthal, MJR Heck, M-C Tien, CM Bruinink, A Leinse and RG Heideman for their useful discussions and expertise.

1 Welch DF, Kish FA, Melle S, Nagarajan R, Kato M et al. Large-scale InP photonic integrated circuits: enabling efficient scaling of optical transport networks. IEEE Select Top Quantum Electron 2007; 13: 22-31.

2 Nicholes SC, Măsanovic ML, Jevremovic B, Lively E, Coldren LA et al. An $8 \times 8 \operatorname{InP}$ monolithic tunable optical router (motor) packet forwarding chip. J Lightwave Technol 2010; 28: 641-50.

3 Liang D, Bowers JE. Photonic integration: Si or InP substrates? Electron Lett 2009; 45: 578-81.

4 Coldren LA, Nicholes SC, Johansson L, Ristic S, Guzzon RS et al. High performance InP-based photonic ICs-a tutorial. J Lightwave Technol 2011; 29: 554-70.

5 Infinera's $400 \mathrm{~Gb} / \mathrm{s}$ PIC sets new record for integration. 23 March 2009. http:// www.infinera.com/j7/servlet/NewsItem?newsItemID $=150$

6 Barwicz T, Watts M, Popovic M, Rakich P, Socci L et al. Polarization-transparent microphotonic devices in the strong confinement limit. Nature Photon 2007; 1 57-60.

7 Sasaki K, Ohno F, Motegi A, Baba T. Arrayed waveguide grating of $70 \times 60 \mu \mathrm{m}^{2}$ size based on Si photonic wire waveguides. Electron Lett 2005; 41: 801-2.

8 Dai D. Liu L, Wosinski L, He S. Design and fabrication of ultra-small overlapped AWG demultiplexer based on alpha-Si nanowire waveguides. Electron Lett 2006; 42: 400 2.

9 Bogaerts W, Selvaraja SK, Dumon P, Brouckaert J, Vos DK et al. Silicon-on-insulator spectral filters fabricated with CMOS technology. IEEE J Sel Top Quant Electron 2010; 16: 33-44.

10 Fukuda H, Yamada K, Tsuchizawa T, Watanabe T, Shinojima $\mathrm{H}$ et al. Silicon photonic circuit with polarization diversity. Opt Express 2008; 16: 4872-80.

11 Sheng Z, Dai D, He S. Comparative study of losses in ultrasharp silicon-on-insulator nanowire bends. IEEE J Sel Top Quant Electron 2009; 15: 1406-112.

12 Liang D, Bowers JE. Recent progress in lasers on silicon. Nature Photon 2010; 4 : 511-7.

13 Alduino A, Liao L, Jones R, Morse M, Kim B et al. Demonstration of a high speed 4channel integrated silicon photonics WDM link with hybrid silicon lasers. OSA technical digest (CD) (Optical Society of America, 2010) in Integrated photonics research, silicon and nanophotonics. Paper PDIWI5

14 Kurczveil G, Heck MJ, Garcia JM, Poulsen HN, Park H et al. Integrated recirculating optical hybrid silicon buffers. SPIE Photonics West; 24 January 2011; San Francisco, CA, USA. SPIE: San Francisco, CA, USA.

15 Faralli S, Nguyen KN, Chen HW, Peters JD, Garcia JM et al. 25 Gbaud DQPSK receiver integrated on the hybrid silicon. Proceedings of 8th IEEE International Conference on Group IV Photonics (GFP); 14-16 September 2011; London, UK, 2011, pp326-8
16 Smit MK, Bente EA, Hill MT, Karouta F, Leijtens XJ et al. Current status and prospects of photonic IC technology. Proceedings of IEEE Conference on Indium Phosphide Related Materials; 14-18 May 2007; Matsue, Japan. IEEE: Matsue, Japan, 2007 pp3-6.

17 Dai D, He S. Design of a polarization-insensitive arrayed waveguide grating demultiplexer based on silicon photonic wires. Opt Lett 2006; 31: 1988-90.

18 Dai D, Shi Y, He S. Theoretical Investigation for reducing polarization-sensitivity in Sinanowire-based arrayed-waveguide grating (de)multiplexer with polarization-beamsplitters and reflectors. IEEE J Quant Electron 2009; 45: 654-60.

19 Pfau T, Peveling R, Hauden J, Grossard N, Porte H et al. Coherent digital polarization diversity receiver for real-time polarization-multiplexed QPSK transmission at $2.8 \mathrm{~Gb}$ s. IEEE Photon Technol Lett 2007; 19: 1988-90.

20 Tien MC, Mizumoto T, Pintus P, Kromer H, Bowers JE. Silicon ring isolators with bonded nonreciprocal magneto-optic garnets. Opt Express 2011; 19: 11740-5.

21 Ciminelli C, Olio FD, Campanella CE, Armenise MN. Photonic technologies for angular velocity sensing. Adv Opt Phot 2010; 2: 370-404.

22 Burmeister EF, Mack JP, Poulsen HN, Mašanovic ML, Stamenic B et al. Photonic integrated circuit optical buffer for packet-switched networks. Opt Express 2009; 17: 6629-35

23 Horikawa K, Ogawa I, Kitoh T, Ogawa H. Silica-based integrated planar lightwave truetime-delay network for microwave antenna applications. Proceedings of the Optica Fiber Communication Conference; 25 February-1 March 1996; San Jose, CA, USA. Optical Society of America: Washington, DC, USA, 1996, Vol. 2, pp100-1.

24 Dong P, Qian W, Liao S, Liang H, Kung CC et al. Low loss shallow-ridge silicon waveguides. Opt Express 2010; 18: 14474-9.

25 Cardenas J, Poitras CB, Robinson JT, Preston K, Chen L et al. Low loss etchless silicon photonic waveguides. Opt Express 2009; 17: 4752-7.

26 Goykhman I, Desiatov B, Levy U. Ultrathin silicon nitride microring resonator for biophotonic applications at $970 \mathrm{~nm}$ wavelength. Appl Phys Lett 2010; 97: 081108.

27 Bogaerts W, Taillaert D, Dumon P, Thourhout DV, Baets R. A polarization-diversity wavelength duplexer circuit in silicon-on-insulator photonic wires. Opt Express 2007; 15: 1567-78.

28 Fukuda H, Yamada K, Tsuchizawa T, Watanabe T, Shinojima $\mathrm{H}$ et al. Ultrasmall polarization splitter based on silicon wire waveguides. Opt Express 2006; 14: 12401-8

29 Komatsu M, Saitoh K, Koshiba M. Design of miniaturized silicon wire and slot waveguide polarization splitter based on a resonant tunneling. Opt Express 2009; 17: 19225-33.

30 Liang TK, Tsang HK. Integrated PBS in high index contrast silicon-on-insulator waveguides. IEEE Photon Technol Lett 2005; 17: 393-5.

31 Augustin LM, Hanfoug R, Tol JJ, Laat WJ, Smit MK, A compact integrated polarization splitter/converter in InGaAsP-InP. IEEE Photon Technol Lett 2007; 19: 1286-8.

32 Hong JM, Ryu HH, Park SR, Jeong JW, Lee SG et al. Design and fabrication of a significantly shortened multimode interference coupler for polarization splitter application. IEEE Photon Technol Lett 2003; 15: 72-4.

33 Jiao $Y$, Dai D, Shi Y, He S. Shortened polarization beam splitters with two cascaded multimode interference sections. IEEE Photon Technol Lett 2009; 21: 1538-40.

34 Tu Z, Huang Y, Yi H, Wang X, Li Y et al. A compact SOI polarization beam splitter based on multimode interference coupler. Asia Communications and Photonics Conference; 13-16 November 2011; Shanghai, China. Paper 8307-06.

35 Yang BK, Shin SY, Zhang DM. Ultrashort polarization splitter using two-mode interference in silicon photonic wires. IEEE Photon Technol Lett 2009; 21: 432-4.

36 Kiyat I, Aydinli A, Dagli N. A compact silicon-on-insulator polarization splitter. IEEE Photon Technol Lett 2005; 17: 100-2.

37 Augustin LM, Tol JJ, Hanfoug RR, de Laat WJ, van de Moosdijk MJ et al. A single etchstep fabrication-tolerant polarization splitter. J Lightwave Technol 2007; 25: 740-6.

38 Xiao J, Liu X, Sun XH. Design of a compact polarization splitter in horizontal multipleslotted waveguide structures. Jpn J App/ Phys 2008; 47: 3748-54.

39 Tu XG, Ang SS, Chew AB, Teng J, Mei T. An ultracompact directional coupler based on GaAs cross-slot waveguide. IEEE Photon Technol Lett 2010; 22: 1324-6.

40 Yamazaki T, Aono H, Yamauchi J, Nakano H. Coupled waveguide polarization splitter with slightly different core widths. J Lightwave Technol 2008; 26: 3528-33.

41 Yue Y, Zhang L, Yang JY, Beausoleil RG, Willner AE. Silicon-on-insulator polarization splitter using two horizontally slotted waveguides. Opt Lett 2010; 35: 1364-1366.

42 Shi Y, Dai D, He S. Proposal for an ultra-compact PBS based on a photonic crystalassisted multimode interference coupler. IEEE Photon Technol Lett 2007; 19(11): 825-7.

43 Ao X, Liu L, Lech W, He S. Polarization beam splitter based on a two-dimensional photonic crystal of pillar type. App/ Phys Lett 2006; 89: 171115.

44 Okuno M, Sugita A, Jinguji K, Kawachi M. Birefringence control of silica waveguides on $\mathrm{Si}$ and its application to a polarization-beam splitter/switch. J Lightwave Technol 1994; 12: 625-34.

45 Goto N, Yip GL. A TE-TM mode splitter in $\mathrm{LiNbO}_{3}$ by proton exchange and Ti diffusion. J Lightwave Technol 1989; 7: 1567-73.

46 Ghirardi F, Brandon J, Carre M, Bruno A, Meniganx L et al. Polarization splitter based on modal birefringence in InP/InGaAsP optical waveguides. IEEE Photon Technol Lett 1993; 5: 1047-9.

47 Lin KC, Chuang WC, Lee WY. Proposal and analysis of an ultrashort directional-couple polarization splitter with an NLC coupling layer. J Lightwave Technol 1996; 14: 2547-53.

48 Dai D, Shi Y, He S. Characteristic analysis of nanosilicon rectangular waveguides for planar light-wave circuits of high integration. App/ Opt 2006; 45: 4941-6. 
49 Almeida VR, Xu Q, Barrios CA, Lipson M. Guiding and confining light in void nanostructure. Opt Lett 2004; 29: 1209-11.

50 Dai D, Wang Z, Bowers JE. Ultra-short broad-band polarization beam splitter based on an asymmetrical directional coupler. Opt Lett 2011; 36: 2590-2.

51 Dai D, Bowers JE. Novel ultra-short and ultra-broadband polarization beam splitter based on a bent directional coupler. Opt Express 2011; 19: 18614-20.

52 Feng NN, Sun R, Kimerling LC, Michel J. Lossless strip-to-slot waveguide transformer. Opt Lett 2007; 32: 1250-2.

53 Lin S, Hu J, Crozier KB. Ultracompact, broadband slot waveguide polarization splitter. Appl Phys Lett 2011; 98: 151101

54 Chin MK, Ho ST. Design and modeling of waveguide-coupled single-mode microring resonators. J Lightwave Technol 1998; 16: 1433-46.

55 Lui WW, Hirono T, Yokoyama K, Huang WP. Polarization rotation in semiconductor bending waveguides: a coupled-mode theory formulation. J Lightwave Technol 1998; 16: 929-36.

56 Zhang J, Yu MB, Lo GQ, Kwong DL. Silicon-waveguide-based mode evolution polarization rotator. IEEE J Select Top Quantum Electron 2010; 16: 53-60.

57 Feng NN, Sun R, Michel J, Kimerling LC. Low-loss compact-size slotted waveguide polarization rotator and transformer. Opt Lett 2007; 32: 2131-3.

58 Yue Y, Zhang L, Song M, Beausoleil RG, Willner AE. Higher-order-mode assisted silicon-on-insulator 90 degree polarization rotator. Opt Express 2009; 17: 20694-9.

59 Rahman BM, Obayya SS, Rajarajan M, Grattan KT, El-Mikathi HA. Design and characterization of compact single-section passive polarization rotator. J Lightwave Technol 2001; 19: 512-9.

60 Deng HH, Yevick DO, Brooks C, Jessop PE. Design rules for slanted-angle polarization rotators. J Lightwave Technol 2005; 23: 432-45.

61 Wang Z, Dai D. Ultrasmall Si-nanowire-based polarization rotator. J Opt Soc Am B 2008; 25: 747-53.

62 Dai D, Bowers JE. Novel concept for ultracompact polarization splitter-rotator based on silicon nanowires. Opt Express 2011; 19: 10940-9.

63 Vermeulen D, Selvaraja S, Cort WA, Yebo NA, Hallynck E. et al.Efficient tapering to the fundamental Quasi-TM mode in asymmetrical waveguides, ECIO 2010; 6-9 April 2010; Cambridge, UK. Department of Information Technology; Cambridge, UK. Paper WeP16.

64 Shintaku T. Integrated optical isolator based on efficient nonreciprocal radiation mode conversion. App/ Phys Lett 1998; 73: 1946-8.

65 Shimizu H, Goto S. Evanescent semiconductor active optical isolators for low insertion loss and high gain saturation power. J Lightwave Technol 2010; 28: 1414-9.

66 Fujita J, Levy M, Osgood RM, Wilkens L, Dotsch H. Waveguide optical isolator based on Mach-Zehnder interferometer. Appl Phys Lett 2000; 76: 2158-60.

67 Yokoi H, Mizumoto T, Shoji Y. Optical nonreciprocal devices with a silicon guiding layer fabricated by wafer bonding. Appl Opt 2003; 42: 6605-12.

68 Yokoi H, Mizumoto T, Shinjo N, Futakuchi N, Nakano Y. Demonstration of an optical isolator with a semiconductor guiding layer that was obtained by use of a nonreciprocal phase shift. Appl Opt 2000; 39: 6158-64

69 Shoji Y, Mizumoto T, Yokoi H, Hsieh IW, Osgood RM. Magneto-optical isolator with silicon waveguides fabricated by direct bonding. App/ Phys Lett 2008; 92: 3.

70 Kono N, Kakihara K, Saitoh K, Koshiba M. Nonreciprocal microresonators for the miniaturization of optical waveguide isolators. Opt Express 2007; 15: 7737-51.

71 Rostami A. Piecewise linear integrated optical device as an optical isolator using twoport nonlinear ring resonators. Opt Laser Technol 2007; 39: 1059-65.

72 Fan L, Wang J, Shen H, Varghese LT, Niu B. et al. A CMOS compatible microring-based on-chip isolator with $18 \mathrm{~dB}$ optical isolation. In: Frontiers in Optics. Rochester: OSA; 2010. pFThQ4.

73 Shintaku T, Uno T, Kobayashi M. Magnetooptic channel waveguides in Ce-substituted yttrium-gartnet. J Appl Phys 1993; 74: 4877-81.

74 Pintus P, Tien MC, Bowers JE. Design of magneto-optical ring isolator on SOI based on the finite-element method, IEEE Photon Technol Lett 2011; 23: 1670-2.

75 Adar R, Serbin MR, Mizrahi V. Less-than-1 dB per meter propagation loss of silica wave-guides measured using a ring-resonator. J Lightwave Technol 1994; 12: 1369_ 72.

76 Kominato T, Hida Y, Itoh M, Takahashi H, Sohma S et al. Extremely low-loss (0.3 dB/ $\mathrm{m})$ and long silica-based waveguides with large width and clothoid curve connection. Proceedings of ECOC; 5-9 September 2004; Stockholm, Sweden, 2004.

77 Bauters JF, Heck MJR, John D, Dai D, Tien MC et al. Ultra-low-loss high-aspect-ratio $\mathrm{Si}_{3} \mathrm{~N}_{4}$ waveguides. Opt Express 2011; 19: 3163-74
78 Dai D, Wang Z, Bauters JF, Tien MC, Heck MJ et al. Low-loss $\mathrm{Si}_{3} \mathrm{~N}_{4}$ arrayed-waveguide grating (de)multiplexer using nano-core optical waveguides. Opt Express 2011; 19: 14130-6.

79 Tien MC, Bauters JF, Heck MJ, Spencer DT, Blumenthal DJ et al. Ultra-high quality factor planar $\mathrm{Si}_{3} \mathrm{~N}_{4}$ ring resonators on Si substrates. Opt Express $2011 ; 19: 13551-6$.

80 Spillane SM, Kippenberg TJ, Vahala KJ. Ultralow-threshold Raman laser using a spherical dielectric microcavity. Nature 2002; 415: 621-3.

81 Vollmer F, Braun D, Libchaber A, Khoshsima M, Teraoka I et al. Protein detection by optical shift of a resonant microcavity. Appl Phys Lett 2002; 80: 4057-9.

82 Klein EJ, Geuzebroek DH, Kelderman H, Gabriel S, Baker N et al. Reconfigurable optical add-drop multiplexer using microring resonators. IEEE Photon Technol Lett 2005; 17: 2358-60.

83 Rasras MS, Kun-Yii T, Gill DM, Young-Kai C, White AE et al. Demonstration of a tunable microwave-photonic notch filter using low-loss silicon ring resonators. J Lightwave Technol 2009; 27: 2105-10.

84 Armani DV, Kippenberg TJ, Spillane SM, Vahala KJ. Ultra-high-Q toroid microcavity on a chip. Nature 2003; 421: 925-8.

85 Soltani M, Yegnanarayanan S, Adibi A. Ultra-high Q planar silicon microdisk resonators for chip-scale silicon photonics. Opt Express 2007; 15: 4694-704.

86 Gondarenko A, Levy JS, Lipson M. High confinement micron-scale silicon nitride high $Q$ ring resonator. Opt Express 2009; 17: 111-5.

87 Hosseini ES, Yegnanarayanan S, Atabaki AH, Soltani M, Adibi A. High quality planar silicon nitride microdisk resonators for integrated photonics in the visible wavelength range. Opt Express 2009; 17: 14543-51.

88 Bauters JF, Heck MJ, John D, Barton JS, Bowers JE et al. Planar waveguides with less than $0.1 \mathrm{~dB} / \mathrm{m}$ propagation loss fabricated with wafer bonding. Opt Express $2011 ; 19$ : 24090-101.

89 Dai D, Wang Z, Bauters JF, Tien MC, Heck MJ et al. Polarization characteristics of lowloss nano-core buried optical waveguides and directional couplers. 7th IEEE International Conference on Group IV Photonics (GFP); 1-3 September 2010; Beijing, China. IEEE Photonics Society, Beijing, China. pp 260-262.

90 Doerr CR, Okamoto K. Advances in silica planar lightwave circuits. J Lightwave Technol 2006; 24: 4763-88.

91 Barbarin Y, Leijtens XJ, Louzao CM, Kooiman JR, Smit MK. Extremely small AWG demultiplexer fabricated on InP by using a double-etch process. IEEE Photon Technol Lett 2004; 16: 2478.

92 Trinh PD, Yegnanarayanan S, Coppinger F, Jalali B. Silicon-on-insulator (SOI) phasedarray wavelength multi/demultiplexer with extremely low-polarization sensitivity. IEEE Photon Technol Lett 1997; 9: 940-2.

93 Dai D, Fu X, Shi Y, He S. Experimental demonstration of an ultra-compact Sinanowire-based reflective arrayed-waveguide grating (de)multiplexer with photonic crystal reflectors. Opt Lett 2010; 35: 2594-6.

94 Dai D, Wang Z, Julian N, Bowers JE. Compact broadband polarizer based on shallowlyetched silicon-on-insulator ridge optical waveguides. Opt Express 2010; 18: 27404 15.

95 Kokubun Y, Asakawa S. ARROW-type polarizer utilizing form birefringence in multilayer first cladding. IEEE Photon Technol Lett 1993; 5: 1418-20.

$96 \mathrm{Li} \mathrm{GY,} \mathrm{Xu} \mathrm{AS.} \mathrm{Analysis} \mathrm{of} \mathrm{the} \mathrm{TE-pass} \mathrm{or} \mathrm{TM-pass} \mathrm{metal-clad} \mathrm{polarizer} \mathrm{with} \mathrm{a} \mathrm{resonant}$ buffer layer. J Lightwave Technol 2008; 26: 1234-41.

97 Twu RC, Huang CC, Wang WS. TE-pass $\mathrm{Zn}$-diffused $\mathrm{LiNbO}_{3}$ waveguide polarizer. Microwav Opt Technol Lett 2006; 48: 2312-4.

98 Cui Y, Wu Q, Schonbrun E, Tinker M, Lee JB et al. Silicon-based 2-D slab photonic crystal TM polarizer at telecommunication wavelength. IEEE Photon Technol Lett 2008; 20: 641-3.

99 Alessandro A, Bellini B, Donisi D, Beccherelli R, Asquini R. Nematic liquid crystal optical channel waveguides on silicon. IEEE J Quant Electron 2006; 42: 1084-90.

100 Wang Q, Ho ST. Ultracompact TM-pass silicon nanophotonic waveguide polarizer and design. IEEE J Photon 2010; 2: 49-56.

(c)

This work is licensed under a Creative Commons Attribution-NonCommercial-NoDerivative Works 3.0 Unported License. To view a copy of this license, visit http:// creativecommons.org/licenses/by-nc-nd/3.0 